\title{
Crustáceos decápodos estuarinos de Ilhéus, Bahia, Brasil
}

\author{
Alexandre Oliveira de Almeida ${ }^{1,2, *}$, Petrônio Alves Coelho ${ }^{2,3}$, José Tiago Almeida dos Santos ${ }^{1}$ \& Neyva Ribeiro Ferraz ${ }^{1}$ \\ Biota Neotropica v6 (n2)-http://www.biotaneotropica.org.br/v6n2/pt/abstract?inventory+bn03406022006 \\ Recebido em 10/11/05 \\ Versão reformulada recebida em 27/04/06 \\ Publicado em 19/07/2006
}

1. Universidade Estadual de Santa Cruz. Departamento de Ciências Biológicas. Rodovia Ilhéus-Itabuna, km 16. $45662-000$ Ilhéus, BA, Brasil (aalmeida@uesc.br)

2. Universidade Federal de Pernambuco. Programa de Pós-Graduação em Oceanografia, Departamento de Oceanografia. Av. da Arquitetura, s/n, Cidade Universitária. 50670-901 Recife, PE, Brasil (petronio.coelho@pesquisador.cnpq.br)

3. Bolsista do CNPq.

*Autor correspondente.

\begin{abstract}
Almeida, A.O., Coelho, P.A., Santos, J.T.A. and Ferraz, N.R. Estuarine Decapod Crustaceans from Ilhéus, State of Bahia, Brazil. Biota Neotrop. May/Aug 2006 vol. 6, no. 2 http://www.biotaneotropica.org.br/v6n2/pt/ abstract?inventory+bn03406022006 ISSN 1676-0603.

The carcinofauna from marine and coastal environments from the southeast and southern Bahia, Brazil, is so far poorly known. The objective of this study was to survey decapod crustaceans occurring along the estuarine environments of Ilhéus, located on the southeast coast of Bahia (1447’55"S, 3902’01"W). Samplings were carried out from February 2001 to February 2005, covering supratidal, intertidal and subtidal habitats. Voucher specimens were fixed in ethanol 70\% and deposited in the collection of Crustacea of the Universidade Estadual de Santa Cruz, Ilhéus (MZUESC). We report a total of 51 species, belonging to 14 families. The most representative family concerning the number of species was Ocypodidae, represented in our samples by 8 species. Geographic range known for the species Alpheus heterochaelis (Caridea: Alpheidae), Merguia rhizophorae (Caridea: Hippolytidae) and Sesarma curacaoense (Brachyura: Sesarmidae) was enlarged. The IndoPacific portunid crab Charybdis hellerii was recorded for the Cachoeira river estuary. The results indicate a high species richness of decapod crustaceans in the estuarine environments of Ilhéus.
\end{abstract}

Key words: crustaceans, diversity, estuaries, geographic distribution, Bahia.

\section{Resumo}

Almeida, A.O., Coelho, P.A., Santos, J.T.A. and Ferraz, N.R. Crustáceos decápodos estuarinos de Ilhéus, Bahia, Brasil. Biota Neotrop. May/Aug 2006 vol. 6, no. 2 http://www.biotaneotropica.org.br/v6n2/pt/ abstract?inventory+bn03406022006 ISSN 1676-0603

A carcinofauna dos ambientes marinhos e costeiros das regiões sudeste e sul da Bahia, Brasil, ainda é pobremente conhecida. O objetivo deste estudo foi realizar um inventário da fauna de crustáceos decápodos do município de Ilhéus, localizado no sudeste da Bahia (1447’55"S, 3902’01"W). As amostragens foram realizadas de Fevereiro de 2001 a Fevereiro de 2005, nas zonas supra, meso e infralitoral. Os exemplares coletados foram fixados em etanol 70\% e depositados na coleção de crustáceos da Universidade Estadual de Santa Cruz, Ilhéus (MZUESC). Foi coletado um total de 51 espécies pertencentes a 14 famílias. A família mais representativa em termos de número de espécies foi Ocypodidae, representada nas amostragens por 8 espécies. A distribuição geográfica conhecida para as espécies Alpheus heterochaelis (Caridea: Alpheidae), Merguia rhizophorae (Caridea: Hippolytidae) e Sesarma curacaoense (Brachyura: Sesarmidae) foi estendida. A ocorrência do siri exótico Charybdis hellerii, originário do Indo-Pacífico, foi registrada para o estuário do rio Cachoeira. Estes resultados indicam que os estuários de Ilhéus abrigam elevada riqueza de espécies.

Palavras-chave: Crustacea, diversidade, estuários, distribuição geográfica, Bahia. 


\section{Introdução}

Um estuário pode ser definido como uma massa de água costeira, semi-fechada, ligada livremente ao mar aberto. Deste modo, é muito influenciado pela ação das marés, havendo uma mistura, em seu interior, da água marinha geralmente bastante diluída com a água doce proveniente das áreas terrestres. Entretanto, muito mais que uma zona de transição entre esses dois habitats, suas características físicas e biológicas principais são, na realidade, exclusivas e não transicionais (Odum 1986).

Crustáceos decápodos são importantes membros das comunidades bentônicas tropicais, incluindo os estuários. Além do fato das espécies maiores e mais abundantes serem usualmente utilizadas como alimento pelo homem, existe uma grande variedade de pequenas espécies que contribuem para o tamanho, complexidade e funcionamento dos ecossistemas tropicais (Hendrickx 1995).

Alguns Estados no Nordeste do Brasil apresentam a fauna de crustáceos decápodos estuarina bem conhecida, com destaque para Pernambuco e Alagoas. Já no Maranhão, Piauí, Ceará e Bahia, o conhecimento sobre a composição da carcinofauna estuarina é incipiente. Diversos trabalhos abordaram a fauna de decápodos dos estuários do Nordeste brasileiro, entre os quais podem ser citados: Rathbun (1900), Coelho (1965/66), Coelho \& Ramos (1972), Ramos-Porto et al (1978), Coelho \& Ramos-Porto (1980), Coelho et al (1986), Coelho \& Coelho-Santos (1990), Coelho et al (1990), CoelhoSantos \& Coelho (2001), Coelho et al (2002), Ferreira \& Sankarankutty (2002) e Calado \& Sousa (2003).

A Bahia é o Estado que possui a maior linha de costa entre os Estados brasileiros (cerca de 1100 km de extensão). Sua rede hidrográfica é rica, destacando-se por sua grande extensão os rios Jequitinhonha, Pardo, Contas, Jiquiriçá e Paraguaçú, entre outros que desembocam no Oceano Atlântico, formando extensas áreas estuarinas. No entanto, os estudos sobre a carcinofauna dessas áreas são muito escassos.

A fauna de crustáceos decápodos dos estuários de Ilhéus é muito pouco conhecida. Williams (1974) assinalou a ocorrência de alguns Portunidae do gênero Callinectes, enquanto Melo (1985) citou algumas espécies de Brachyura para a região. O presente trabalho teve como objetivo determinar a composição da fauna de crustáceos decápodos dos estuários de Ilhéus. Espera-se que estes dados possam servir de base para a realização de estudos sobre a ecologia e biologia da carcinofauna local, para a elaboração de planos de manejo e utilização sustentada das espécies.

\section{Material e Métodos}

\section{Descrição da Área de Estudo}

O município de Ilhéus, situado na região sudeste da Bahia (1447’55"S; 3902’01"W) (Figura 1), abrange uma área de $1712 \mathrm{~km}^{2}$. Possui um vasto litoral, com cerca de $80 \mathrm{~km}$ de extensão, limitado ao norte pelo rio Sargi e ao sul pelo rio Acuípe.

O clima caracteriza-se pelos tipos úmido e subúmido. As temperaturas médias anuais variam entre $22^{\circ} \mathrm{C} \mathrm{e} 25^{\circ} \mathrm{C}$. A pluviosidade apresenta totais anuais superiores a $1000 \mathrm{~mm}$, chegando a alcançar 2700 mm em locais próximos ao litoral. O regime pluviométrico é regular, com chuvas abundantes, distribuídas durante o ano (Faria-Filho \& Araújo 2003).

As áreas de manguezal mais representativas de Ilhéus localizam-se na zona urbana, ao longo das margens da porção estuarina dos rios Cachoeira, Santana, Fundão e Almada (P.I.J. Fidelman, comunicação pessoal) (Figuras 2-5).

O maior estuário é o do rio Cachoeira, que desemboca em meio à área urbana do município, banhando o centro da cidade e o bairro do Pontal (Figura 2). Juntamente com os rios Fundão e Santana forma um complexo estuarino. Os principais tensores ambientais são os efluentes domésticos do município de Itabuna, a leste de Ilhéus, bem como efluentes domésticos e resíduos sólidos do bairro Teotônio Vilela no próprio município. Grandes extensões de seus manguezais têm sido aterradas ao longo da BR-415 (P.I.J. Fidelman, comunicação pessoal).

O rio Almada forma o segundo maior estuário da região, tendo sua foz ao norte da cidade de Ilhéus (Figura 3). Os principais tensores ambientais são os efluentes domésticos e resíduos sólidos do bairro São Domingos (P.I.J. Fidelman, comunicação pessoal).

Entre os rios de pequeno porte destacam-se o Sargi e o Mamoã, no litoral norte, e o Cururupe e o Acuípe, ao sul. O estuário do Cururupe encontra-se bastante alterado, pois até o início desta década suas margens abrigavam o lixão da cidade. Os estuários dos demais rios caracterizam-se pela reduzida influência antrópica e consequentemente encontram-se melhor preservados.

\section{Métodos de Amostragem}

As amostragens foram qualitativas, sem padronização de esforço. Foram amostradas as zonas supra, meso e infralitoral.

No supra e mesolitoral, as coletas foram realizadas no período entre Fevereiro de 2001 e Fevereiro de 2005, sendo mais intensas a partir de Fevereiro de 2003. A freqüência das amostragens não foi pré-determinada. Estuários maiores, como os dos rios Almada, Cachoeira, Santana e Acuípe, foram visitados em diversas ocasiões, enquanto que os menores estuários dos rios Sargi, Mamoã e Cururupe foram visitados uma única vez (coordenadas geográficas dos pontos de amostragem no anexo). Também foi amostrado o manguezal do ribeirão Iguape, que deságua no rio Fundão, no Parque Municipal da Boa Esperança. No estuário do rio 

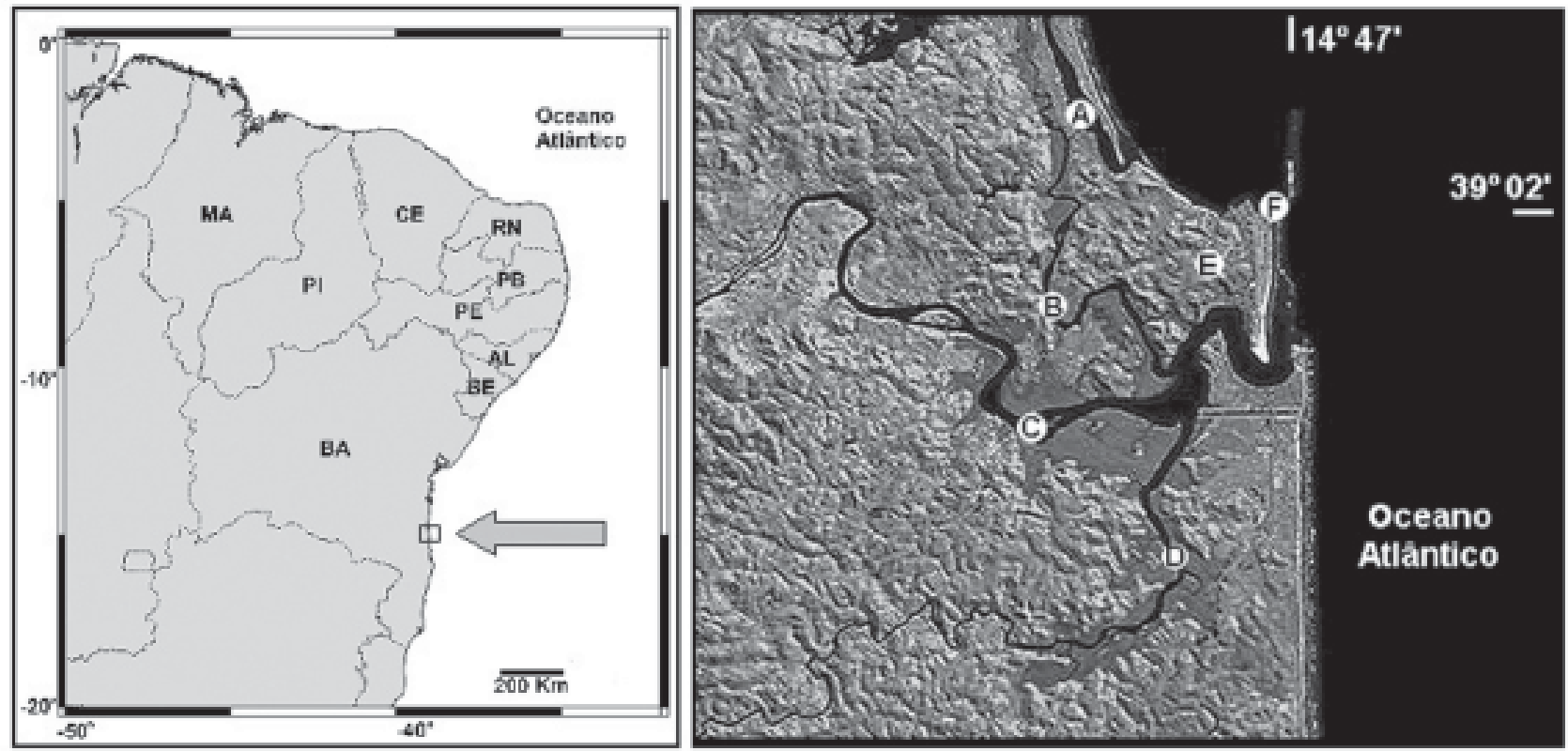

Figura 1. Localização da área de estudo, Ilhéus, Bahia, Brasil. (A) Rio Almada, (B), rio Fundão, (C) rio Cachoeira, (D) rio Santana, (E) área central da cidade de Ilhéus, (F) porto de Ilhéus. Fonte: Miranda \& Coutinho (2004).

Figure 1. Localization of the study area: Ilhéus, State of Bahia, Brazil. (A) Almada River, (B), Fundão River, (C) Cachoeira River, (D) Santana River, (E) Ilhéus central area, (F) Ilhéus harbor. Source: Miranda \& Coutinho (2004).

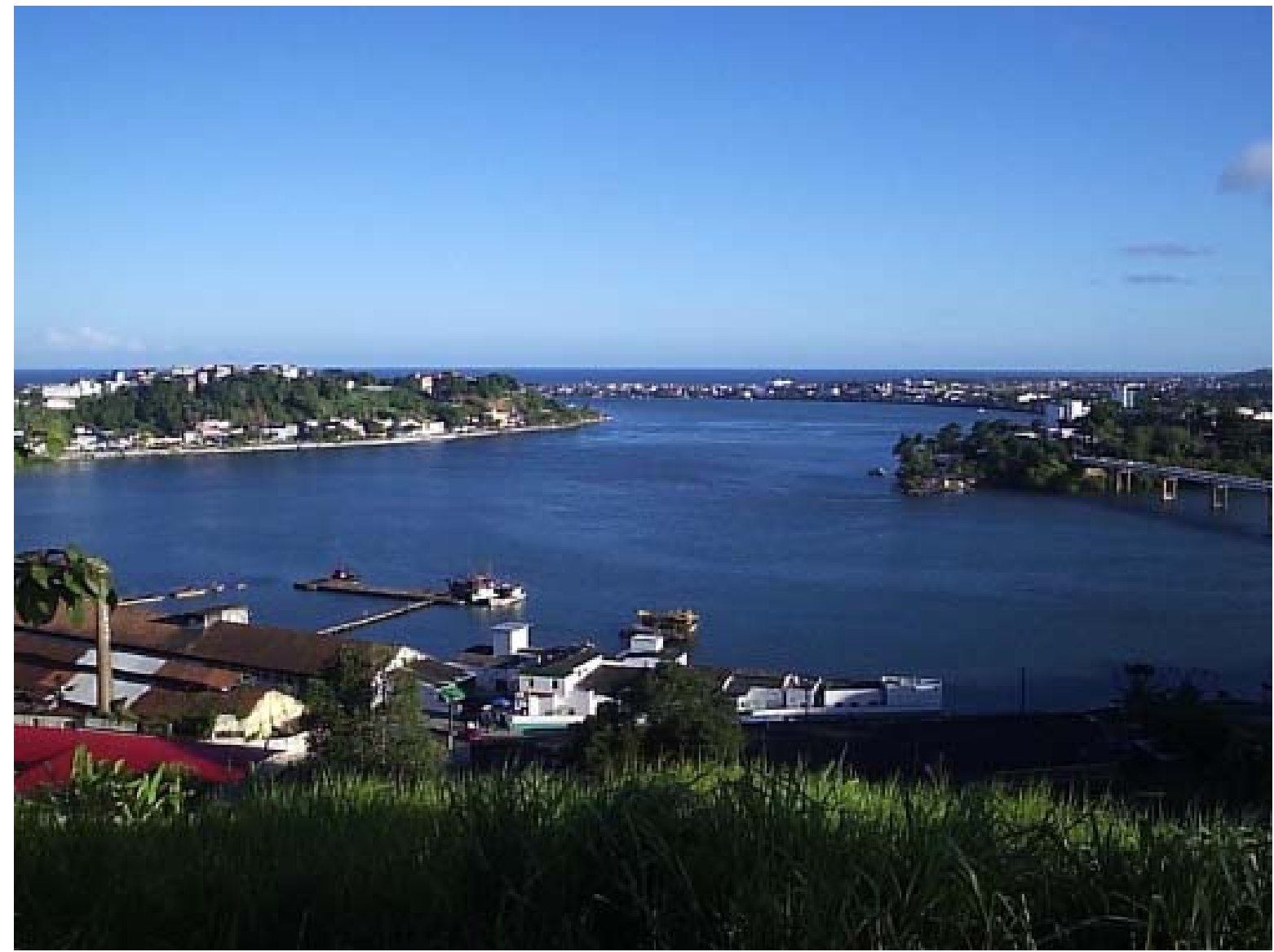

Figura 2. Estuário do rio Cachoeira, Ilhéus, Brasil: o maior estuário da região.

Figure 2. Cachoeira River estuary, Ilhéus, Brazil: the largest estuary of the region. 


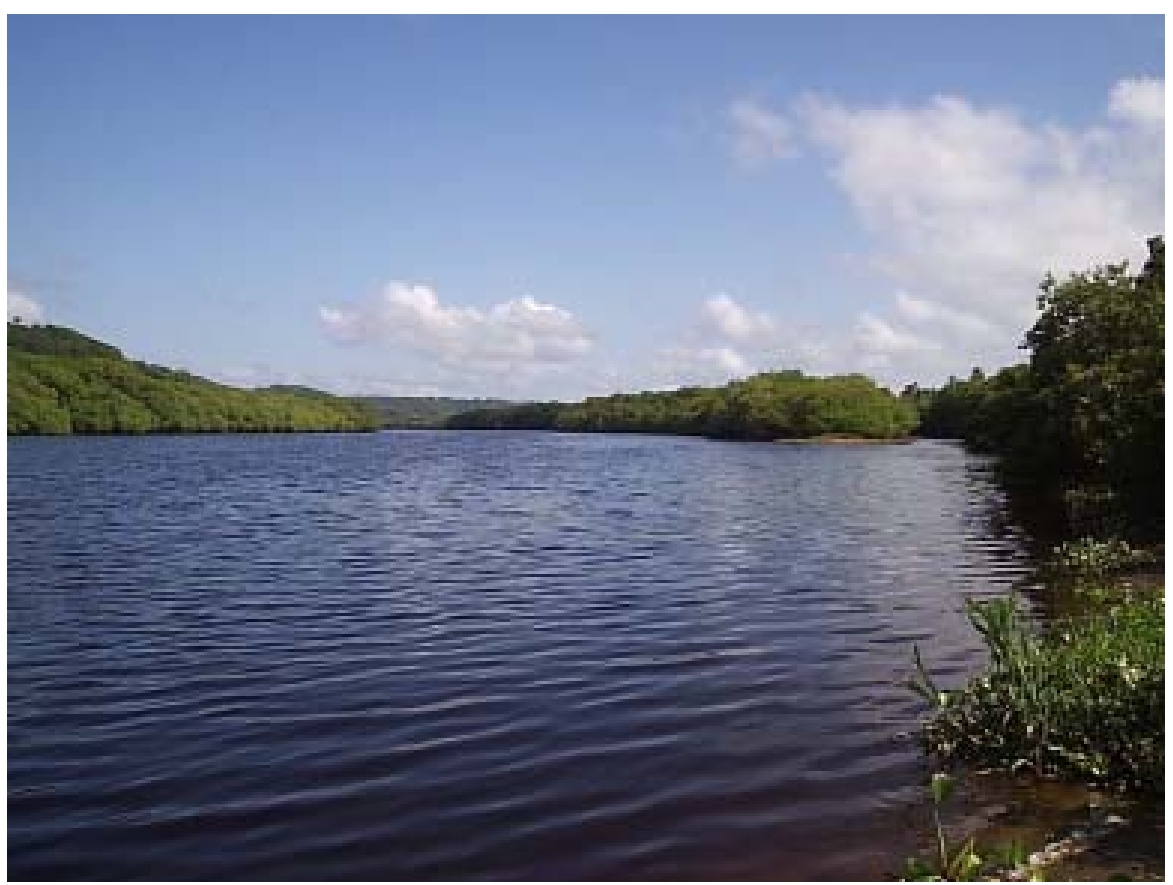

Figura 3. Manguezal do estuário do rio Almada, Ilhéus, Brasil.

Figure 3. The mangrove of the Almada River, Ilhéus, Brazil.

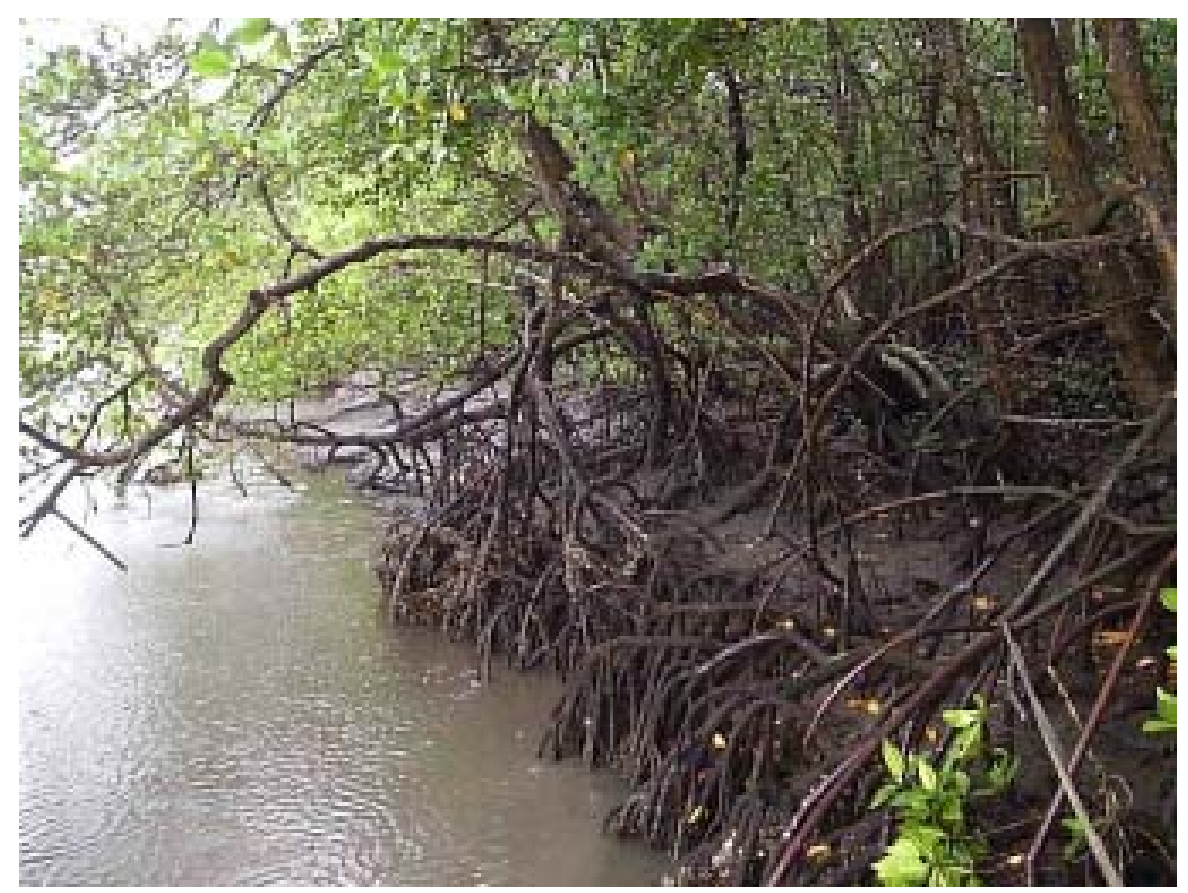

Figura 4. Manguezal com Rhizophora mangle (Rhizophoraceace) no estuário do rio Santana, Ilhéus, Brasil. Figure 4. Rhizophora mangle (Rhizophoraceace) from the Santana River estuary, Ilhéus, Brazil. 


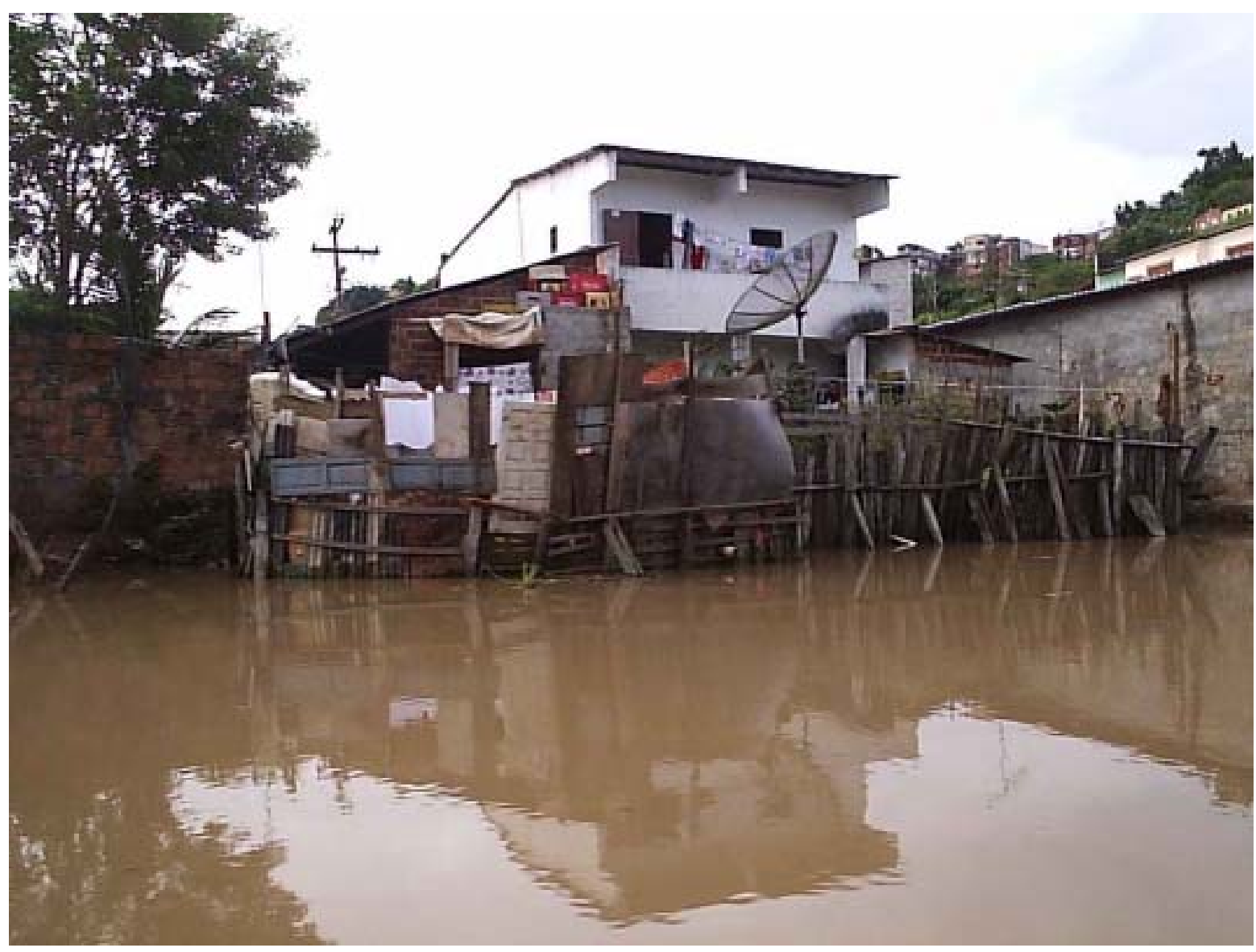

Figura 5. Impacto da urbanização descontrolada em um canal estuarino próximo ao centro da cidade de Ilhéus, Brasil. Figure 5. Uncontrolled urbanization in an estuarine channel near Ilhéus central area, Brazil.

Cachoeira foram realizadas coletas inclusive na área urbana, como as avenidas Sapetinga, 2 de Julho, Lomanto Júnior, praia do Cristo e praia da Maramata (ver coordenadas geográficas no anexo). As coletas efetuadas durante a baixamar foram realizadas manualmente e com o auxílio de armadilhas artesanais como a siripóia (equipamento artesanal para pesca de siris). Procurou-se abranger todos os microhabitats do ambiente estuarino, tais como tocas escavadas na areia e lama, folhas e troncos em processo de decomposição, raízes e troncos das árvores do mangue, sob e sobre pedras, em bancos de algas, mexilhões e ostras.

O infralitoral foi amostrado através de barco de arrasto, num total de 4 amostragens, que foram realizadas entre Setembro de 2004 e Janeiro de 2005, com barco de pesca camaroneiro, equipado com rede trainete, para amostragens de fundo. A rede era equipada com um par de portas e mediu entre 4 e $5 \mathrm{~m}$ de comprimento de total e $40 \mathrm{~cm}$ de altura. A malha da rede, no corpo, mediu entre 18 e $20 \mathrm{~mm}$ entre nós, e no sacador 15 mm entre nós. Foi amostrado o infralitoral dos rios Santana e Cachoeira, do ponto máximo navegável à montante, até a foz.
Os espécimes capturados foram fixados em álcool etílico 70\% e depositados na coleção de crustáceos do Museu de Zoologia da Universidade Estadual de Santa Cruz (MZUESC). Para cada espécie é fornecida a descrição do material examinado, a distribuição geográfica e notas ecológicas. Foram incluídas fotografias das três espécies cuja distribuição geográfica foi aumentada até Ilhéus. O número entre parênteses situado ao lado de material examinado representa o $\mathrm{N}$ total de indivíduos examinados de cada espécie. A classificação até o nível de família adotada neste trabalho segue a proposta por Martin \& Davis (2001).

\section{Resultados}

Foi coletado um total de 1282 espécimes, pertencentes a 51 espécies e 14 famílias. A subordem Dendrobranchiata esteve representada por juvenis da família Penaeidae. A subordem Pleocyemata esteve representada por 4 infraordens: Caridea - famílias Palaemonidae (quatro espécies), Alpheidae (sete espécies) e Hippolytidae (uma espécie); Thalassinidea - famílias Callianassidae (uma espécie) e Upogebiidae (uma espécie); Anomura - famílias 
Tabela 1 - Lista de espécies de crustáceos decápodos e sua distribuição nos estuários de Ilhéus, Bahia, Brasil $(\bullet=$ material coletado e depositado na coleção MZUESC; 0 ocorrência constatada em campo; - ocorrência não constatada).

Table 1 - Decapod species from Ilhéus, State of Bahia, Brazil: list of species and distribution per estuary $(\bullet=$ specimens deposited in Crustacea collection at MZUESC; 0 field observation; - species neither collected nor observed).

\begin{tabular}{|c|c|c|c|c|c|c|c|c|}
\hline TAXA & $\begin{array}{l}\text { P. M. Boa } \\
\text { Esperança }\end{array}$ & $\begin{array}{c}\text { Rio } \\
\text { Acuípe }\end{array}$ & $\begin{array}{c}\text { Rio } \\
\text { Almada }\end{array}$ & $\begin{array}{c}\text { Rio } \\
\text { Cachoeira }\end{array}$ & $\begin{array}{c}\text { Rio } \\
\text { Cururupe }\end{array}$ & $\begin{array}{c}\text { Rio } \\
\text { Mamoã }\end{array}$ & $\begin{array}{c}\text { Rio } \\
\text { Santana }\end{array}$ & $\begin{array}{c}\text { Rio } \\
\text { Sargi }\end{array}$ \\
\hline \multicolumn{9}{|l|}{ PENAEIDAE } \\
\hline Farfantepenaeus sp & - & - & - & $\bullet$ & - & - & $\bullet$ & - \\
\hline \multicolumn{9}{|l|}{ PALAEMONIDAE } \\
\hline Macrobrachium & - & $\bullet$ & $\bullet$ & - & - & - & - & - \\
\hline \multicolumn{9}{|l|}{ acanthurus } \\
\hline Leander paulensis & - & - & - & $\bullet$ & - & - & $\bullet$ & - \\
\hline Palaemon northropi & - & - & $\bullet$ & $\bullet$ & - & - & - & - \\
\hline Palaemon pandaliformis & - & $\bullet$ & - & - & - & - & - & - \\
\hline \multicolumn{9}{|l|}{ ALPHEIDAE } \\
\hline Alpheus armillatus & - & - & $\bullet$ & $\bullet$ & - & - & - & - \\
\hline Alpheus bouvieri & - & - & - & $\bullet$ & - & - & - & - \\
\hline Alpheus estuariensis & - & - & - & - & - & $\bullet$ & - & - \\
\hline Alpheus heterochaelis & - & - & - & $\bullet$ & - & - & $\bullet$ & - \\
\hline Alpheus intrinsecus & - & - & - & $\bullet$ & - & - & - & - \\
\hline Alpheus pontederiae & $\bullet$ & $\bullet$ & - & $\bullet$ & - & - & - & - \\
\hline Synalpheus fritzmuelleri & - & - & - & $\bullet$ & - & - & - & - \\
\hline \multicolumn{9}{|l|}{ HIPPOLYTIDAE } \\
\hline Merguia rhizophorae & - & - & - & - & - & $\bullet$ & - & - \\
\hline \multicolumn{9}{|l|}{ CALLIANASSIDAE } \\
\hline Lepidophtalmus siriboia & - & - & - & $\bullet$ & - & - & - & - \\
\hline \multicolumn{9}{|l|}{ UPOGEBIIDAE } \\
\hline Upogebia brasiliensis & - & - & - & $\bullet$ & $\bullet$ & - & $\bullet$ & - \\
\hline \multicolumn{9}{|l|}{ PORCELLANIDAE } \\
\hline Petrolisthes armatus & - & - & - & $\bullet$ & - & - & - & - \\
\hline \multicolumn{9}{|l|}{ DIOGENIDAE } \\
\hline Clibanarius antillensis & - & - & - & $\bullet$ & - & - & - & - \\
\hline Clibanarius sclopetarius & - & - & $\bullet$ & $\bullet$ & - & - & - & $\bullet$ \\
\hline Clibanarius vittatus & - & - & $\bullet$ & $\bullet$ & - & - & - & - \\
\hline \multicolumn{9}{|l|}{ PORTUNIDAE } \\
\hline Callinectes bocourti & - & - & - & $\bullet$ & - & - & $\bullet$ & - \\
\hline Callinectes danae & - & $\bullet$ & $\bullet$ & $\bullet$ & - & - & $\bullet$ & $\bullet$ \\
\hline Callinectes exasperatus & - & $\bullet$ & $\bullet$ & $\bullet$ & - & $\bullet$ & $\bullet$ & \\
\hline
\end{tabular}


Continuação

\begin{tabular}{|c|c|c|c|c|c|c|c|c|}
\hline TAXA & $\begin{array}{l}\text { P. M. Boa } \\
\text { Esperança }\end{array}$ & $\begin{array}{c}\text { Rio } \\
\text { Acuípe }\end{array}$ & $\begin{array}{c}\text { Rio } \\
\text { Almada }\end{array}$ & $\begin{array}{c}\text { Rio } \\
\text { Cachoeira }\end{array}$ & $\begin{array}{c}\text { Rio } \\
\text { Cururupe }\end{array}$ & $\begin{array}{c}\text { Rio } \\
\text { Mamoã }\end{array}$ & $\begin{array}{c}\text { Rio } \\
\text { Santana }\end{array}$ & $\begin{array}{c}\text { Rio } \\
\text { Sargi }\end{array}$ \\
\hline Callinectes larvatus & - & - & - & $\bullet$ & - & - & - & $\bullet$ \\
\hline Callinectes ornatus & - & - & - & $\bullet$ & - & - & - & - \\
\hline Callinectes sapidus & - & - & - & $\bullet$ & - & - & - & - \\
\hline Charybdis hellerii & - & - & - & $\bullet$ & - & - & - & - \\
\hline \multicolumn{9}{|l|}{ PANOPEIDAE } \\
\hline Acatholobulus schmitti & - & - & - & $\bullet$ & - & - & - & - \\
\hline \multicolumn{9}{|l|}{ abbreviatus } \\
\hline Eurytium limosum & - & $\bullet$ & $\bullet$ & - & $\bullet$ & $\bullet$ & - & $\bullet$ \\
\hline $\begin{array}{l}\text { Hexapanopeus } \\
\text { caribbaeus }\end{array}$ & \multicolumn{7}{|c|}{ caribbaeus } & - \\
\hline Panopeus lacustris & - & $\bullet$ & $\bullet$ & $\bullet$ & $\bullet$ & $\bullet$ & $\bullet$ & - \\
\hline Panopeus occidentalis & - & $\bullet$ & - & $\bullet$ & - & $\bullet$ & $\bullet$ & - \\
\hline \multicolumn{9}{|l|}{ OCYPODIDAE } \\
\hline Ocypode quadrata & - & 0 & ० & 0 & ० & $\bullet$ & - & $\bullet$ \\
\hline Uca leptodactyla & $\bullet$ & $\bullet$ & $\bullet$ & $\bullet$ & $\bullet$ & $\bullet$ & - & $\bullet$ \\
\hline Uca maracoani & - & $\bullet$ & $\bullet$ & - & - & - & - & - \\
\hline Uca mordax & - & - & $\bullet$ & - & - & - & - & - \\
\hline Uca rapax & $\bullet$ & $\bullet$ & $\bullet$ & - & - & $\bullet$ & $\bullet$ & - \\
\hline Uca thayeri & $\bullet$ & $\bullet$ & $\bullet$ & - & $\bullet$ & $\bullet$ & $\bullet$ & $\bullet$ \\
\hline Uca vocator & - & - & $\bullet$ & - & - & - & - & - \\
\hline \multicolumn{9}{|l|}{ GECARCINIDAE } \\
\hline \multicolumn{9}{|l|}{ GRAPSIDAE } \\
\hline Cyclograpsus integer & - & - & - & - & - & $\bullet$ & - & - \\
\hline Goniopsis cruentata & $\bullet$ & $\bullet$ & $\bullet$ & $\bullet$ & $\bullet$ & $\bullet$ & $\bullet$ & $\bullet$ \\
\hline Pachygrapsus gracilis & $\bullet$ & $\bullet$ & $\bullet$ & $\bullet$ & $\bullet$ & $\bullet$ & $\bullet$ & $\bullet$ \\
\hline $\begin{array}{l}\text { Pachygrapsus } \\
\text { transversus }\end{array}$ & - & - & $\bullet$ & $\bullet$ & - & - & - & - \\
\hline \multicolumn{9}{|l|}{ SESARMIDAE } \\
\hline Aratus pisonii & $\bullet$ & $\bullet$ & $\bullet$ & $\bullet$ & - & $\bullet$ & - & $\bullet$ \\
\hline Armases angustipes & $\bullet$ & $\bullet$ & $\bullet$ & - & - & $\bullet$ & - & - \\
\hline Armases rubripes & $\bullet$ & - & $\bullet$ & - & - & $\bullet$ & - & - \\
\hline Sesarma curacaoense & - & $\bullet$ & $\bullet$ & - & $\bullet$ & $\bullet$ & - & - \\
\hline Sesarma rectum & $\bullet$ & $\bullet$ & $\bullet$ & - & - & - & - & - \\
\hline
\end{tabular}

http://www.biotaneotropica.org.br 
Porcellanidae (uma espécie) e Diogenidae (três espécies); Brachyura - famílias Portunidae (sete espécies), Panopeidae (sete espécies), Ocypodidae (oito espécies), Gecarcinidae (uma espécie), Grapsidae (quatro espécies) e Sesarmidae (cinco espécies). Informações sobre a distribuição das espécies por estuário encontram-se na tabela 1 . A lista de espécies por estação de coleta encontra-se no anexo.

\section{Ordem Decapoda Latreille, 1802}

Subordem Dendrobranchiata Bate, 1888

Superfamília Penaeoidea Rafinesque, 1815

Família Penaeidae Rafinesque, 1815

\section{Farfantepenaeus sp.}

Material examinado ( $\mathrm{N}=18)$ : $\mathrm{N}=1,18 / 09 / 2004$, rio Santana, Est. 03(MZUESC 622); N=1, 18/09/2004, rio Santana, Est. 04 (MZUESC 623); N=2, 18/09/2004, rio Cachoeira, Est. 06 (MZUESC 624); N=2, 18/09/2004, rio Cachoeira, Est. 08 (MZUESC 625); N=1, 24/09/2004, rio Cachoeira, Est. 03 (MZUESC 626); N=4, 24/09/2004, rio Cachoeira, Est. 05 (MZUESC 627); N=2, 24/09/2004, rio Cachoeira, Est. 06 (MZUESC 628); $\mathrm{N}=1,13 / 01 / 2005$, rio Cachoeira, Est. 04 (MZUESC 629); N=3, 13/01/2005, rio Cachoeira, Est. 06 (MZUESC 630); N=1, 13/01/2005, rio Cachoeira, Est. 08 (MZUESC 631).

Comentários: Foram citadas três espécies deste gênero para águas costeiras da região: F. subtilis (Pérez-Farfante, 1967) e F. brasiliensis (Latreille, 1817), por Coelho \& Santos (1995), e F. paulensis (Pérez-Farfante, 1967), por D’Incao (1995). Os indivíduos coletados nos estuários eram todos juvenis, não tendo sido possível a identificação até o nível de espécie.

\section{Subordem Pleocyemata Burkenroad, 1963 \\ Infraordem Caridea Dana, 1852}

Superfamília Palaemonoidea Rafinesque, 1815

Família Palaemonidae Rafinesque, 1815

Macrobrachium acanthurus (Wiegmann, 1836)

Material examinado (N=3): N=2, 01/05/2003, rioAcuípe, Est. 03 (MZUESC 106); N=1, 22/02/2005, rio Almada, Est. 04 (MZUESC 560).

Distribuição: Da Carolina do Norte ao Texas, México, Cuba, Haiti, República Dominicana, Porto Rico, Nicarágua, Panamá, Colômbia, Venezuela, Suriname e Brasil (do Pará ao Rio Grande do Sul) (Melo 2003).

Notas ecológicas: Águas escuras, geralmente paradas, com vegetação emergente e substrato lodoso. Às vezes encontrado em águas salobras (Melo 2003). Indivíduos juvenis foram coletados no manguezal do rio Acuípe, na baixa-mar, em poças entre raízes de Rhizophora mangle (Rhizophoraceae). O exemplar coletado no estuário do rio Almada, já adulto, encontrava-se associado às raízes de "baronesa”, Eicchornia crassipes (Pontederiaceae), sendo sua ocorrência possivelmente acidental.

\section{Leander paulensis Ortmann, 1897}

Material examinado ( $\mathrm{N}=15)$ : $\mathrm{N}=2,18 / 09 / 2004$, rio Santana, Est. 04 (MZUESC 581); N=4, 18/09/2004, rio Cachoeira, Est. 07 (MZUESC 586); N=5, 18/09/2004, rio Cachoeira, Est. 08 (MZUESC 587); N=1, 13/01/2005, rio Santana, Est. 04 (MZUESC 607); N=1, 13/01/2005, rio Cachoeira, Est. 07 (MZUESC 613); N=2, 13/01/2005, rio Cachoeira, Est. 08 (MZUESC 617).

Distribuição: Atlântico Ocidental - Flórida, Antilhas e Brasil (Maranhão, Ceará, Paraíba Pernambuco, Bahia e São Paulo) (Ramos-Porto 1985/86).

Notas ecológicas: Ocorre em fundos de areia, com prados de algas moles e fanerógamas marinhas. De águas rasas até 16 m (Ramos-Porto 1985/86).

Palaemon (Palaeander) northropi (Rankin, 1898)

Material examinado ( $\mathrm{N}=19)$ : $\mathrm{N}=7,21 / 08 / 2004$, rio Cachoeira, praia da Maramata (MUESC 419); N=12, 22/02/ 2005, rio Almada, Est. 02 (MZUESC 550).

Distribuição: Atlântico Ocidental - Bermuda, Antilhas, América Central, norte da América do Sul, Brasil (do Ceará até Santa Catarina) e Uruguai (Ramos-Porto \& Coelho 1990).

Notas ecológicas: No entre-marés e infralitoral raso. Em ambiente marinho e estuarino, em fundos arenosos e rochosos costeiros (Fausto Filho 1978, Fausto Filho 1979, Ramos-Porto \& Coelho 1990). Todos os espécimes foram coletados em poças de maré. 1871)

Palaemon (Palaemon) pandaliformis (Stimpson,

Material examinado (N=4): N=4, 01/05/2003, rioAcuípe, Est. 03 (MZUESC 90).

Distribuição: Cuba, Porto Rico, Barbados, Trinidad e Tobago, Guatemala, Nicarágua, Panamá, Venezuela e Brasil (Rio Grande do Norte e de Sergipe ao Rio Grande do Sul, incluindo Minas Gerais) (Melo 2003).

Notas ecológicas: Espécie de águas doces e ambientes estuarinos, encontrada com freqüência junto às raízes da vegetação aquática (Bond-Buckup \& Buckup 1989). Indivíduos foram coletados durante a baixa-mar, em poças formadas entre raízes de Rhizophora mangle.

\section{Superfamília Alpheoidea Rafinesque, 1815 \\ Família Alpheidae Rafinesque, 1815 \\ Alpheus armillatus H. Milne Edwards, 1837}

Material examinado ( $\mathrm{N}=12)$ : $\mathrm{N}=6,22 / 02 / 2005$, rio Almada, Est. 02 (MZUESC 551); N=4, 24/09/2004, rio Cachoeira, Est. 08 (MZUESC 598); N=2, 13/01/2005, rio Cachoeira, Est. 08 (MZUESC 618). 
Distribuição: Atlântico Ocidental - Bermuda, Carolina do Norte, Flórida, Golfo do México, Antilhas, Venezuela e Brasil (Fernando de Noronha e do Ceará até Santa Catarina) (Martínez-Iglesias et al 1996, Christoffersen 1998).

Notas ecológicas: Em baías e pequenos ambientes protegidos da ação direta das ondas, sendo comum em estuários. Em fundos lamosos, de areia, em bancos de Thalassia (Hydrocharitaceae) e algas verdes, sob pedras e conchas, em recifes de coral. De águas rasas até $14 \mathrm{~m}$ (Martínez-Iglesias et al 1996, Calado \& Sousa 2003).

Alpheus bouvieri A. Milne-Edwards, 1878

Material examinado $(\mathrm{N}=4)$ : $\mathrm{N}=4,21 / 08 / 2004$, rio Cachoeira, praia da Maramata (MZUESC 495).

Distribuição: Atlântico Ocidental - Bermuda, Flórida, Cuba, Antígua a Trinidad e Tobago, Aruba e Brasil (Fernando de Noronha e do Ceará ao Rio Grande do Sul). Atlântico Oriental - Cabo Verde, do Senegal a São Tomé e Príncipe, Congo. Pacífico Leste - da Costa Rica ao Equador, Galápagos (Christoffersen 1979, Martínez-Iglesias et al 1996).

Notas ecológicas: Ocorre na zona entre-marés, entre pedras ou associado a colônias de Phragmatopoma (Sabellariidae) e esponjas (Christoffersen 1979).

\section{Alpheus estuariensis Christoffersen, 1984}

Material examinado (N=7): N=7, 21/01/2005, rio Mamoã (MZUESC 530).

Distribuição: Atlântico Ocidental - Flórida, do Mississippi ao Texas, Cuba, República Dominicana, Trinidad e Tobago, Curaçao e Brasil (do Ceará ao Paraná). Pacífico Leste - Baía Concepción (Golfo da Califórnia), Costa Rica, Panamá, Baía Málaga (Colômbia) (Christoffersen 1984, Martínez-Iglesias et al 1996).

Notas ecológicas: Espécie restrita a ambientes estuarinos. Fundos de areia e lama, associado a rochas, ostras e raízes do mangue, do entre-marés até 22 m de profundidade (Christoffersen 1984).

\section{Alpheus heterochaelis Say, 1818 (Figura 6)}

Material examinado ( $\mathrm{N}=8)$ : $\mathrm{N}=4,18 / 09 / 2004$, rio Santana, Est. 03 (MZUESC 580); N=4, 24/09/2004, rio Cachoeira, Est. 04 (MZUESC 592).

Distribuição: Atlântico Ocidental - da Carolina do Norte até o Brasil (Alagoas) (Christoffersen 1984, Calado \& Sousa 2003).

Notas ecológicas: Ocorre em habitações no substrato lodoso ou areno-lodoso, habitualmente associada a bancos de ostras, pedras e troncos em decomposição, sendo uma típica espécie estuarina. Do entre-marés até $9 \mathrm{~m}$ de profundidade (Christoffersen 1984, Calado \& Sousa 2003).

\section{Alpheus intrinsecus Bate, 1888}

Material examinado ( $\mathrm{N}=5)$ : $\mathrm{N}=3,18 / 09 / 2004$, rio Cachoeira, Est. 06 (MZUESC 584); N=2, 13/01/2005, rio Cachoeira, Est. 08 (MZUESC 619).

Distribuição: Atlântico Ocidental - de Porto Rico ao Brasil (do Piauí até Santa Catarina). Atlântico Oriental - do Saara Ocidental ao Gabão (Christoffersen 1979).

Notas ecológicas: Lama, argila, areia, areia e gorgônias, associado à Halodule (Cymodoceaceae), areia grossa e conchas e algas calcáreas. Do entre-marés até $40 \mathrm{~m}$ (Christoffersen 1979). Espécie coletada em estações situadas próximas à boca do estuário do rio Cachoeira.

\section{Alpheus pontederiae Rochebrune, 1883}

Material examinado $(\mathrm{N}=10)$ : $\mathrm{N}=6,01 / 05 / 2003$, rio Acuípe, Est. 02 (MZUESC 86); N=1, 21/05/2003, Pque Mun. Boa Esperança (MZUESC 125); N=1, 04/07/2003, rio Acuípe, Est. 05 (MZUESC 203); N=2, 24/09/2004, rio Cachoeira, Est. 04(MZUESC 593).

Distribuição:Atlântico Ocidental Brasil (Pará, Maranhão, Alagoas, São Paulo, Paraná). Atlântico Oriental - do Senegal ao Zaire (Christoffersen 1984, Calado \& Sousa 2003).

Notas ecológicas: Em troncos de madeira morta, vegetação aquática ou em habitações na areia fina e lama. Ambiente estuarino, do entre-marés até 30 m (Christoffersen 1984, Calado \& Sousa 2003).

\section{Synalpheus fritzmuelleri Coutière, 1909}

Material examinado $(\mathrm{N}=3): \mathrm{N}=3,24 / 09 / 2004$, rio Cachoeira, Est. 08 (MZUESC 599).

Distribuição: Atlântico Ocidental - Bermuda, Carolinas, Flórida, Texas, Vera Cruz (Golfo do México), Quintana Roo (México), Ilha de Providência, Jamaica, Porto Rico até Trinidad e Aruba, Venezuela e Brasil (Pernambuco até Santa Catarina). Atlântico Central - Ilha de Santa Helena. Pacífico Leste Arquipélago Três Marias (México) (Christoffersen 1979).

Notas ecológicas: Em esponjas, entre ascídias, em colônias de Zoanthus (Zoanthidae), gorgônias, raízes de árvores do manguezal, associada à Halimeda opuntia (Udoteaceae), algas calcáreas, colônias de Schizoporella (Schizoporellidae) e Phragmatopoma, corais, cavidades de rochas e rochas calcáreas. Do entre-marés até 75 m (Christoffersen 1979). Os exemplares examinados foram coletados em estação situada próxima à boca do estuário do rio Cachoeira.

Família Hippolytidae Dana, 1852

Merguia rhizophorae (Rathbun, 1900) (Figura 7)

Material examinado ( $\mathrm{N}=13)$ : $\mathrm{N}=13,21 / 01 / 2005$, rio Mamoã (MZUESC 529). 


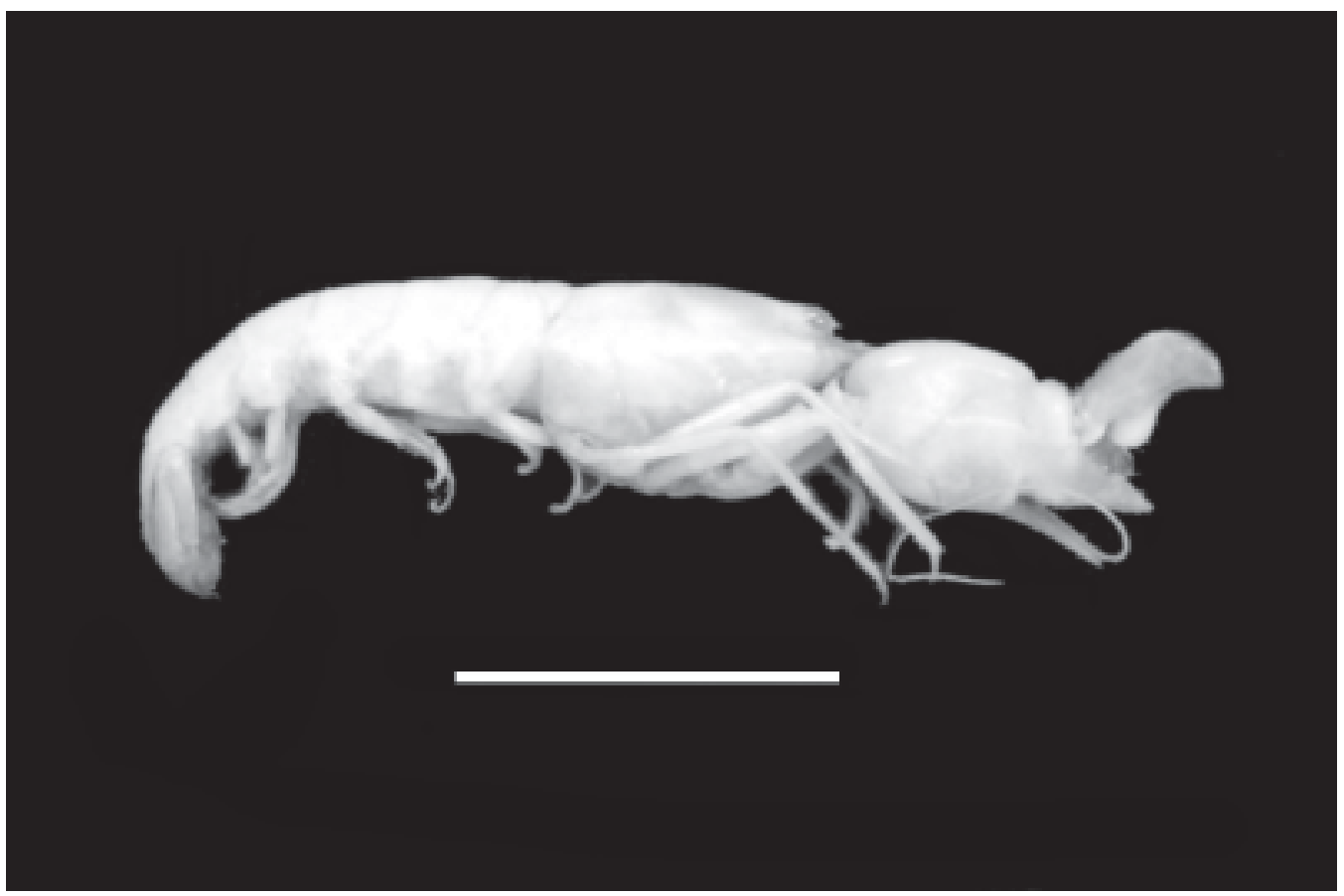

Figura 6. Alpheus heterochaelis Say do estuário do rio Cachoeira, Ilhéus, Brasil. Barra = $10 \mathrm{~mm}$

Figure 6. Alpheus heterochaelis Say from the Cachoeira River estuary, Ilhéus, Brazil. Scale bar $=10 \mathrm{~mm}$.

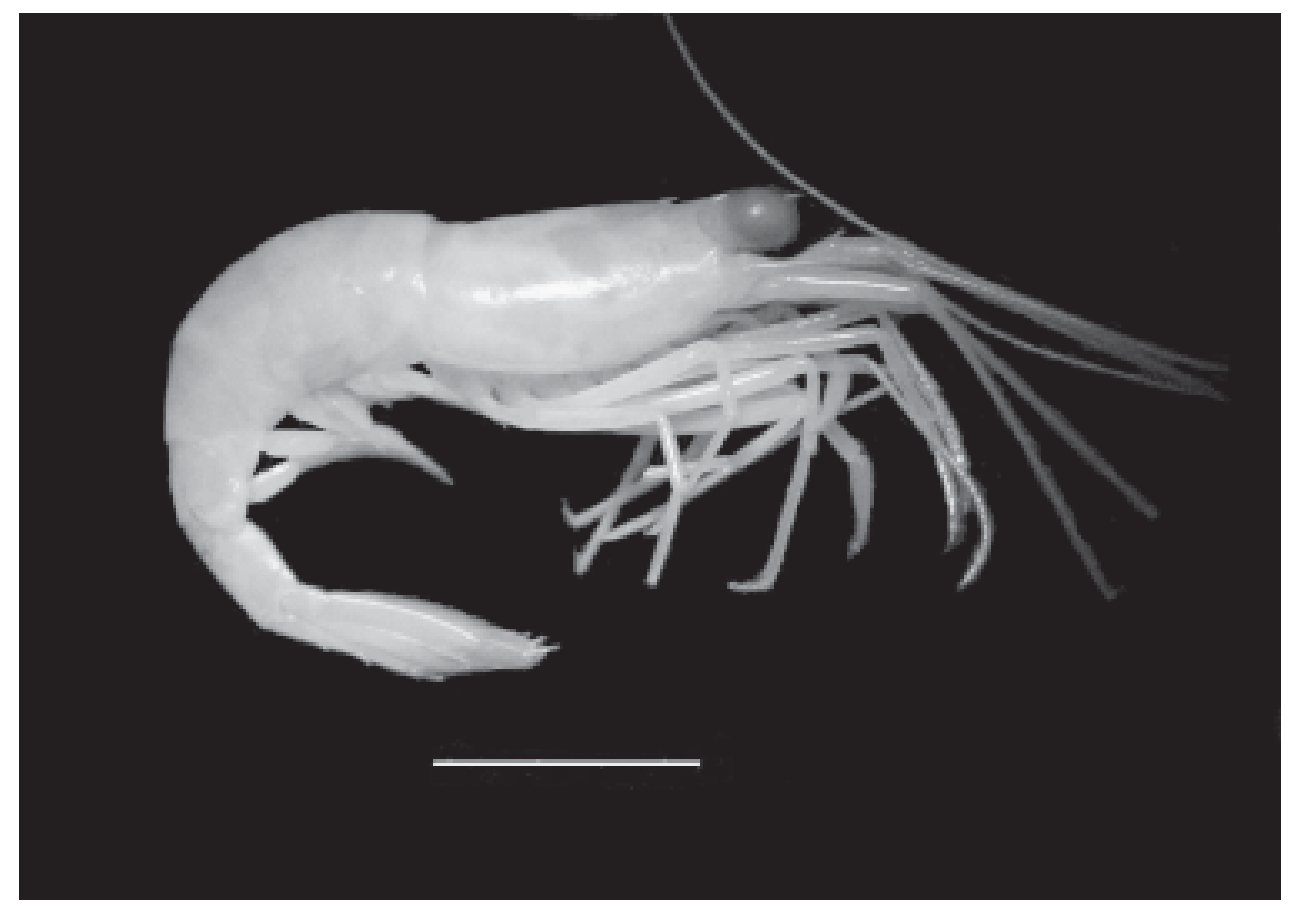

Figura 7. Merguia rhizophorae Rathbun do estuário do rio Mamoã, Ilhéus, Brasil. Barra = $5 \mathrm{~mm}$

Figure 7. Merguia rhizophorae Rathbun from the Mamoã River estuary, Ilhéus, Brazil. Scale bar $=5 \mathrm{~mm}$.

http://www.biotaneotropica.org.br 
Distribuição: Atlântico Ocidental - Panamá, Suriname e Brasil (do Piauí a Alagoas) (Chace Jr 1972, Christoffersen 1998, Calado \& Sousa 2003).

Notas ecológicas: Na região entre-marés, em águas rasas e no interior de troncos em decomposição. Ocupa habitats descobertos durante a baixa-mar (Calado \& Sousa 2003). A espécie foi coletada apenas no manguezal do rio Mamoã, durante a baixa-mar, no interior de troncos de $R$. mangle .

\section{Infraordem Thalassinidea Latreille, 1831 \\ Superfamília Callianassoidea Dana, 1852}

Família Callianassidae Dana, 1852

Lepidophtalmus siriboia Felder \& Rodrigues, 1993

Material examinado ( $\mathrm{N}=2$ ): $\mathrm{N}=2,26 / 05 / 2004$, rio Cachoeira, praia da Maramata (MZUESC 414).

Distribuição: Atlântico Ocidental - Flórida, Golfo do México, Antilhas e Brasil (do Pará até a Bahia) (Melo 1999).

Notas ecológicas: Águas rasas, em galerias submersas na região do entre-marés (Melo 1999). Os exemplares examinados foram coletados próximo à desembocadura do rio Cachoeira.

Família Upogebiidae Borradaile, 1903

Upogebia brasiliensis Holthuis, 1956

Material examinado ( $\mathrm{N}=6)$ : $\mathrm{N}=1,16 / 07 / 2003$, rio Cururupe (MZUESC 218); N=1, 18/09/2004, rio Santana, Est. 03 (MZUESC 498); N=4, 24/09/2004, rio Cachoeira, Est. 08 (MZUESC 600).

Distribuição: Atlântico Ocidental - Belize, Guiana Francesa, Suriname e Brasil (do Pará até Santa Catarina) (Melo 1999).

Notas ecológicas: Em buracos, sob pedras e pedaços de madeira no entre-marés de praias areno-lodosas (Melo 1999). Os espécimes foram coletados no interior de troncos em decomposição.

\section{Infraordem Anomura MacLeay, 1838}

Superfamília Galatheoidea Samouelle, 1819

Família Porcellanidae Haworth, 1825

Petrolisthes armatus (Gibbes, 1850)

Material examinado ( $\mathrm{N}=21)$ : $\mathrm{N}=21,21 / 08 / 2004$, rio Cachoeira, praia da Maramata (MZUESC 417).

Distribuição: Atlântico Ocidental - da Carolina do Norte à Flórida, Golfo do México, Antilhas, Colômbia e Brasil (Fernando de Noronha, e do Pará até Santa Catarina). Atlântico Oriental - do Senegal até Angola. Atlântico Central - Ilha Ascensão. Pacífico Leste - Golfo da Califórnia, Costa Rica, Equador (Galápagos e Peru) (Barros et al 1997b, Melo 1999).
Notas ecológicas: Sob pedras, em corais, bancos de mexilhões, esponjas, ostras e em raízes de Rhizophora e Avicennia (Verbenaceae). Do entre-marés aos $60 \mathrm{~m}$ (Melo 1999). Espécimes coletados durante a baixa-mar, na desembocadura do rio Cachoeira.

\section{Superfamília Paguroidea Latreille, 1802}

Família Diogenidae Ortmann, 1892

Clibanarius antillensis Stimpson, 1859

Material examinado ( $\mathrm{N}=5$ ): $\mathrm{N}=5,21 / 08 / 2004$, rio Cachoeira, praia da Maramata (MZUESC 492).

Distribuição: Atlântico Ocidental - Bermuda, Flórida, Golfo do México, Panamá, Antilhas, norte da América do Sul e Brasil (Atol das Rocas e do Ceará até Santa Catarina) (Melo 1999).

Notas ecológicas: No entre-marés, em cavidades de rochas e também em arrecifes e prados de Halodule (Melo 1999). Coletada sob pedras, durante a baixa-mar, na desembocadura do rio Cachoeira.

\section{Clibanarius sclopetarius (Herbst, 1796)}

Material examinado ( $\mathrm{N}=33)$ : $\mathrm{N}=6,13 / 03 / 2003$, rio Cachoeira, praia do Cristo (MZUESC 36); N=4, 13/03/2003, rio Cachoeira, Av. 02 de Julho (MZUESC 43); N=3, 03/03/ 2004, rio Sargi (MZUESC 325); N=11, 21/08/2004, rio Cachoeira, praia da Maramata (MZUESC 490); N=8, 22/02/ 2005, rio Almada, Est. 02 (MZUESC 549); N=1, 13/01/2005, rio Cachoeira, Est. 06 (MZUESC 610).

Distribuição: Atlântico Ocidental - Flórida, Antilhas, Venezuela, norte da América do Sul, Guianas e Brasil (do Ceará até Santa Catarina) (Melo 1999).

Notas ecológicas: Na região entre-marés, em fundos de areia, em estuários e recifes. Ocasionalmente em prados de Halodule e Halophila (Hydrocharitaceae) (Melo 1999).

\section{Clibanarius vittatus (Bosc, 1802)}

Material examinado ( $\mathrm{N}=19)$ : $\mathrm{N}=3,13 / 03 / 2003$, rio Cachoeira, praia do Cristo (MZUESC 37); N=2, 21/08/2004, rio Cachoeira, praia da Maramata (MZUESC 491); $\mathrm{N}=11$, 22/ 02/2005, rio Almada, Est. 02 (MZUESC 555); N=1, 18/09/ 2004, rio Cachoeira, Est. 06 (MZUESC 585); N=1, 18/09/2004, rio Cachoeira, Est. 08 (MZUESC 590); N=1, 13/01/2005, rio Cachoeira, Est. 06 (MZUESC 609).

Distribuição: Atlântico Ocidental - costa leste dos EUA, Golfo do México, Antilhas, Venezuela, Suriname e Brasil (do Pará até Santa Catarina) (Melo 1999).

Notas ecológicas: Estuários, recifes de coral, em fundos de areia e prados de Halodule. De águas rasas até 22 m (Melo 1999). 


\section{Infraordem Brachyura Latreille, 1802}

Seção Eubrachyura de Saint Laurent, 1980 Superfamília Portunoidea Rafinesque, 1815

Família Portunidae Rafinesque, 1815

Callinectes bocourti A. Milne Edwards, 1879

Material examinado $(\mathrm{N}=8)$ : $\mathrm{N}=2,18 / 09 / 2004$, rio Santana, Est. 01 (MZUESC 422); N=3, 24/09/2004, rio Cachoeira, Est. 03 (MZUESC 438); N=1, 13/01/2005, rio Cachoeira, Est. 01 (MZUESC 461); N=1, 13/01/2005, rio Santana, Est. 04(MZUESC 468); N=1, 13/01/2005, rio Santana, Est. 04 (MZUESC 469).

Distribuição: Atlântico Ocidental - costa leste dos EUA, Antilhas, norte da América do Sul e Brasil (do Amapá até o Rio Grande do Sul) (Melo1996, Santos et al 2000).

Notas ecológicas: Espécie estuarina, que prefere águas com baixa salinidade, também presente em águas poluídas. Do entre-marés aos 20 m (Melo 1996).

Comentários: Espécie citada previamente para Ilhéus por Williams (1974) e Melo (1985).

\section{Callinectes danae Smith, 1869}

Material examinado ( $\mathrm{N}=127)$ : $\mathrm{N}=1,20 / 02 / 2001$, rio Cachoeira, Av. Sapetinga (MZUESC 02); N=10, 13/03/2003, rio Cachoeira, praia do Cristo (MZUESC 39); $\mathrm{N}=8$, 23/04/ 2003, rio Acuípe, Est. 06, foz (MZUESC 71); N=4, 01/05/ 2003, rio Acuípe, Est. 02 (MZUESC 78); N=13, 03/03/2004, rio Sargi (MZUESC 320); N=4, 04/03/2004, rio Acuípe, Est. 04 (MZUESC 336); N=6, 14/11/2003, rio Almada (MZUESC 371); $\mathrm{N}=5,04 / 12 / 2003$, rio Almada (MZUESC 372); $\mathrm{N}=2$, 18/ 09/2004, rio Cachoeira, Est. 08 (MZUESC 420); N=1, 18/09/ 2004, rio Cachoeira, Est. 06 (MZUESC 421); N=2, 18/09/2004, rio Santana, Est. 04 (MZUESC 425); N=2, 24/09/2004, rio Cachoeira, Est. 06 (MZUESC 434); N=2, 24/09/2004, rio Cachoeira, Est. 02 (MZUESC 435); N=7, 24/09/2004, rio Cachoeira, Est. 03 (MZUESC 437); N=6, 24/09/2004, rio Cachoeira, Est. 05 (MZUESC 439); N=13, 24/09/2004, rio Cachoeira, Est. 04 (MZUESC 454); N=1, 13/01/2005, rio Cachoeira, Est. 06 (MZUESC 457); N=1, 13/01/2005, rio Cachoeira, Est. 03 (MZUESC 458); N=5, 13/01/2005, rio Cachoeira, Est. 06 (MZUESC 459); N=1, 13/01/2005, rio Cachoeira, Est. 05 (MZUESC 460); N=3, 13/01/2005, rio Cachoeira, Est. 02 (MZUESC 462); N=4, 13/01/2005, rio Cachoeira, Est. 07 (MZUESC 464); N=3, 13/01/2005, rio Santana, Est. 04 (MZUESC 466); N=9, 13/01/2005, rio Cachoeira, Est. 06 (MZUESC 467); N=6, 22/02/2005, rio Fundão, Est. 01 (MZUESC 547); N=7, 22/02/2005, rioAlmada, Est. 02 (MZUESC 554); N=1, 13/01/2005, rio Cachoeira, Est. 07 (MZUESC 615).

Distribuição: Atlântico Ocidental - Bermuda, Flórida, Golfo do México, Antilhas, Colômbia, Venezuela e Brasil (do Pará ao Rio Grande do Sul) (Melo 1996, Barros et al 1997a).
Notas ecológicas: Ocorre em águas salobras até hipersalinas, em manguezais e estuários lamosos. Também em praias arenosas e mar aberto, do entre marés até 75 m (Melo 1996).

Comentários: Espécie citada previamente para Ilhéus por Williams (1974) e Melo (1985). Pela representatividade em nossas amostragens, a espécie parece ser a mais abundante entre os Portunidae da região.

\section{Callinectes exasperatus (Gerstaecker, 1856)}

Material examinado ( $\mathrm{N}=28)$ : $\mathrm{N}=3,13 / 03 / 2003$, rio Cachoeira, Av. 02 de Julho (MZUESC 40); N=2, 20/02/2001, rio Cachoeira, Av. Sapetinga (MZUESC 47); N=1, 04/07/2003, rio Acuípe, Est. 05 (MZUESC 202); N=2, 18/09/2004, rio Santana, Est. 04 (MZUESC 424); N=11, 13/01/2005, rio Cachoeira, Est. 05 (MZUESC 456); N=1, 21/01/2005, rio Mamoã (MZUESC 531); N=7, 22/02/2005, rio Almada, Est. 02 (MZUESC 553); N=1, 13/01/2005, rio Cachoeira, Est. 06 (MZUESC 611).

Distribuição: Atlântico Ocidental - Bermuda, Flórida, Golfo do México, Antilhas, Colômbia, Venezuela e Brasil (do Pará até Santa Catarina) (Melo 1996, Barros \& Pimentel 2001).

Notas ecológicas: Pode ser encontrado em águas rasas, do entre-marés até $8 \mathrm{~m}$. Em águas salgadas e estuarinas perto de bocas de rios e de manguezais (Melo 1996).

Comentários: Espécie citada previamente para Ilhéus por Williams (1974) e Melo (1985).

\section{Callinectes larvatus Ordway, 1863}

Material examinado ( $\mathrm{N}=22)$ : $\mathrm{N}=7,20 / 02 / 2001$, rio Cachoeira, praia da Maramata (MZUESC 01); N=2, 20/02/ 2001, rio Cachoeira, Av. Sapetinga (MZUESC 05); N=3, 13/ 03/2003, rio Cachoeira, Av. 02 de Julho (MZUESC 41); N=3, 13/03/2003, rio Cachoeira, Av. 02 de Julho (MZUESC 42); $\mathrm{N}=3,03 / 03 / 2004$, rio Sargi (MZUESC 319); $N=4,21 / 08 / 2004$, rio Cachoeira, praia da Maramata (MZUESC 493).

Distribuição: Atlântico Ocidental - da Carolina do Norte até a Flórida, Golfo do México, Bermuda, Antilhas, Colômbia, Venezuela e Brasil (do Pará até São Paulo) (Melo 1996, Barros et al 1997a).

Notas ecológicas: Em fundos de areia e lama, periferia de manguezais, também em água salobra, raramente em mar aberto. Do entre-marés até 25 m (Melo 1996). Notou-se preferência desta espécie por áreas situadas próximas à desembocadura dos rios.

Comentários: Espécie citada previamente para Ilhéus por Williams (1974), como C. marginatus, e por Melo (1985).

\section{Callinectes ornatus Ordway, 1863}

Material examinado ( $\mathrm{N}=2)$ : $\mathrm{N}=1,18 / 09 / 2004$, rio Cachoeira, Est. 08 (MZUESC 423); N=1, 13/01/2005, rio Cachoeira, Est. 07 (MZUESC 463). 
Distribuição: Atlântico Ocidental - Carolina do Norte até a Flórida, Golfo do México, Antilhas, Colômbia, Venezuela, Guianas e Brasil (do Amapá até o Rio Grande do Sul) (Melo 1996).

Notas ecológicas: Em fundos de areia e lama, até os 75 m de profundidade (Melo 1996). A espécie foi coletada em estações próximas à desembocadura do rio Cachoeira.

\section{Callinectes sapidus Rathbun, 1895}

Material examinado ( $\mathrm{N}=2$ ): $\mathrm{N}=1,24 / 09 / 2004$, rio Cachoeira, Est. 05 (MZUESC 433); N=1, 24/09/2004, rio Cachoeira, Est. 02 (MZUESC 436).

Distribuição: Atlântico Ocidental - costa leste dos EUA, Antilhas, América Central, Venezuela e Brasil (de Alagoas até o Rio Grande do Sul). Atlântico Oriental, Mar Mediterrâneo, Adriático e Negro. Pacífico Oeste - Japão (Melo 1996, Calado 2000).

Notas ecológicas: Em estuários, lagunas, baías e água doce (Melo 1996).

Charybdis hellerii A. Milne Edwards, 1867 (Figura 8)

Material examinado $(\mathrm{N}=1)$ : $\mathrm{N}=1,18 / 01 / 2005$, rio Cachoeira, Est. 07 (MZUESC 614).

Distribuição: Mar Vermelho, Djibouti, Somália, África do Sul, Madagascar, Golfo Pérsico, Hong Kong, Cingapura, Ceilão, Índia, China, Japão, Indonésia, Filipinas, Nova Caledônia, Austrália e Havaí. Mediterrâneo Oriental - Egito e Israel. Atlântico Ocidental - Flórida, Cuba, Colômbia, Venezuela, Guiana Francesa e Brasil (do Ceará até Santa Catarina) (Dineen et al 2001, Tavares \& Amouroux 2003, Bezerra \& Almeida 2005).

Notas ecológicas: Preferência por sedimentos não consolidados, podendo, entretanto, ser encontrada em sedimentos consolidados, como rochas e corais. Do entremarés até $51 \mathrm{~m}$ de profundidade (Lemaitre 1995).

Comentários: Espécie exótica, de origem IndoPacífica, registrada pela primeira vez na costa de Bahia por Carqueija \& Gouvêa (1996), na Baía de Todos os Santos, litoral norte. Posteriormente, Almeida et al (2003) registraramna para a Baía de Camamu. Um único exemplar foi coletado com rede de arrasto no estuário do rio Cachoeira, representando o terceiro registro de C. hellerii para costa da Bahia.

Características do espécime coletado (Figura 8): Forma da carapaça de hexagonal a retangular. Tamanho 3x4 mm (comprimento de caraparaça x largura de carapaça). Apêndices ambulatórios perdidos. Margem lateral da carapaça dotada de seis dentes ântero-laterais conspícuos e fronte com seis dentes rudimentares. Morfologia da carapaça está de acordo com a do terceiro estágio juvenil (ver Dineen et al 2001, figura 24C).
A espécie em questão poderá ser distinguida dos demais Portunidae estuarinos nativos através da seguinte chave, adaptada de Melo (1996):

(1) Carapaça com seis dentes ântero-laterais, incluindo o orbital externo. Charybdis hellerii

(1') Carapaça com nove dentes ântero-laterais, incluindo o orbital externo. ...2

(2) Fronte com dois dentes sub-triangulares de base alargada, situados entre os espinhos orbitais internos... Callinectes sapidus

(2') Fronte com quatro dentes entre os espinhos orbitais internos.. 3

(3) Dentes frontais pouco diferentes em tamanho. Dente lateral da carapaça pouco desenvolvido quando comparado aos demais dentes ânterolaterais. .4

(3’) Dentes frontais de tamanho desigual, tendo os dois internos menos da metade do comprimento dos dois externos. Dente lateral da carapaça bastante desenvolvido quando comparado aos demais dentes ânterolaterais. .5

(4) Regiões ântero-laterais da carapaça lisas ao tato. Gonópodos do macho adulto atingindo a extremidade do abdome... .Callinectes bocourti

(4’) Regiões ântero-laterais da carapaça ásperas tato. Gonópodos do macho adulto atingindo a metade do penúltimo segmento do abdome Callinectes exasperatus

(5) Dentes ântero-laterais, com exceção dos dois primeiros, com ápice curvado para frente. Gonópodo do macho adulto bastante curto. .Callinectes larvatus

(5’) Apenas o último, ou os dois últimos dentes ânterolaterais com ápice curvado para frente. 6

(6) Par interno de dentes sub-mesiais frontais pouco desenvolvidos. Carapaça com área central trapezoidal com largura aproximada de 3 vezes o seu comprimento.....................................Callinectes ornatus

(6’) Par interno de dentes sub-mesiais frontais desenvolvidos. Carapaça com área central trapezoidal com largura aproximada de 2,5 vezes o seu comprimento. ..Callinectes danae

\section{Superfamília Xanthoidea MacLeay, 1838}

Família Panopeidae Ortmann, 1893

Acantholobulus schmitti (Rathbun, 1930)

Material examinado ( $\mathrm{N}=1)$ : $\mathrm{N}=1,24 / 09 / 2004$, rio Cachoeira, Est. 08 (MZUESC 601).

Distribuição: Atlântico Ocidental - Brasil (do Ceará até Santa Catarina) e Uruguai (Melo 1996). 


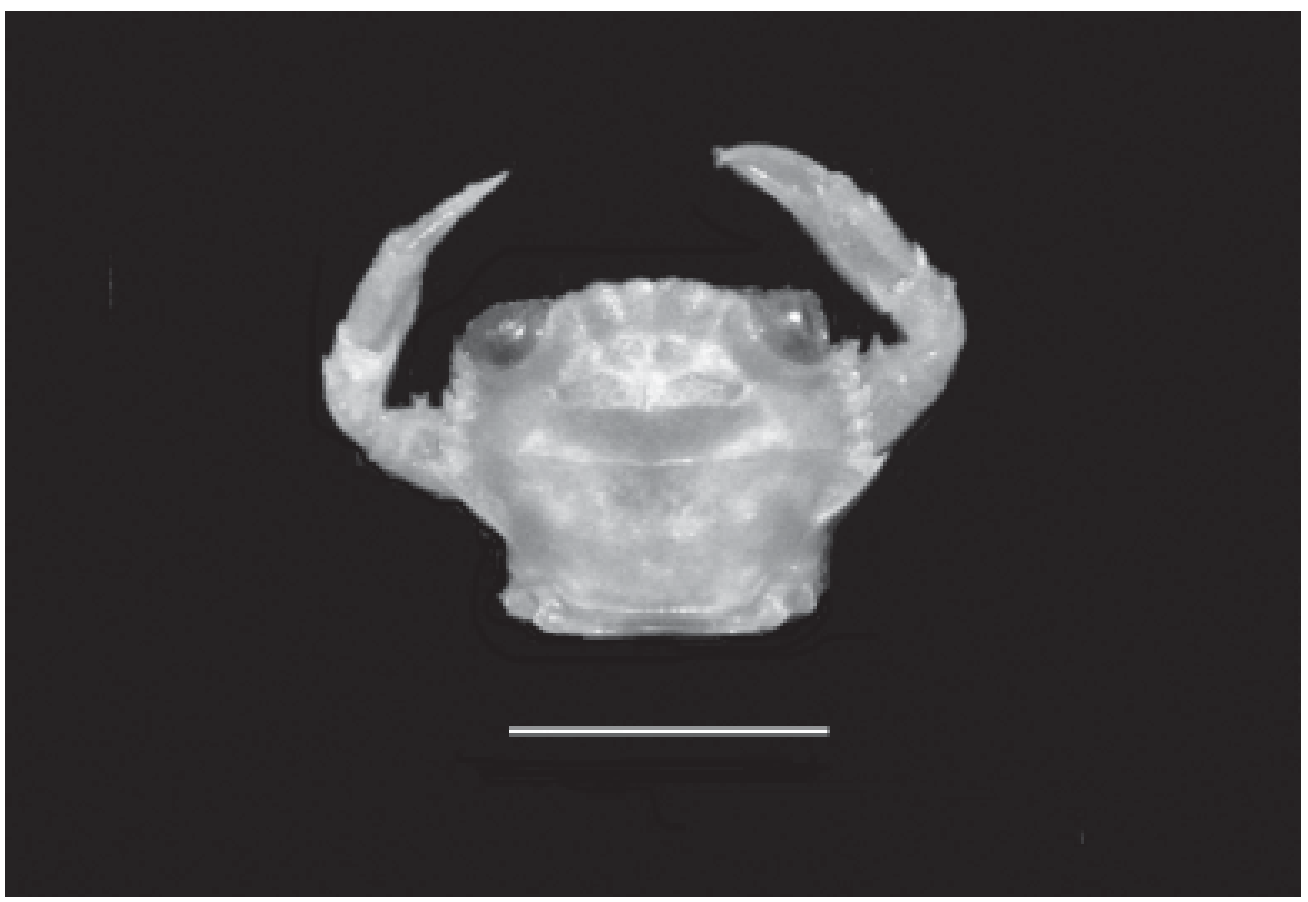

Figura 8. Juvenil de Charybdis hellerii A. Milne Edwards do estuário do rio Cachoeira, Ilhéus, Brasil. Barra = $3 \mathrm{~mm}$.

Figure 8. Juvenile of Charybdis hellerii A. Milne Edwards from the Cachoeira River estuary, Ilhéus, Brazil. Scale bar = $3 \mathrm{~mm}$.

Notas ecológicas: Ocorre em fundos de areia, lama e conchas, desde o entre-marés até 25 m (Melo 1996).

\section{Eurypanopeus abbreviatus (Stimpson, 1860)}

Material examinado $(\mathrm{N}=3)$ : $\mathrm{N}=3,21 / 08 / 2004$, rio Cachoeira, praia da Maramata (MZUESC 633).

Distribuição: Atlântico Ocidental - costa leste dos EUA, Golfo do México, Antilhas, norte da América do Sul e Brasil (do Ceará ao Rio Grande do Sul) (Melo 1996).

Notas ecológicas: Em fundos rochosos e de coral, desde o entre-marés até 5 m (Melo 1996). Foi coletada junto à foz do rio Cachoeira.

\section{Eurytium limosum (Say, 1818)}

Material examinado $(\mathrm{N}=16)$ : $\mathrm{N}=5,01 / 05 / 2003$, rio Acuípe, Est. 02 (MZUESC 82); N=1, 04/06/2003, rio Acuípe, Est. 05 (MZUESC 149); N=1, 04/07/2003, rio Acuípe (MZUESC 235); $\mathrm{N}=1,16 / 07 / 2003$, rio Cururupe (MZUESC 237); $N=1,14 / 11 / 2003$, rio Almada (MZUESC 253); $N=1,03 /$ 03/2004, rio Sargi (MZUESC 324); $N=1,04 / 12 / 2003$, rio Almada (MZUESC 398); N=1, 16/07/2003, rio Cururupe (MZUESC 401); N=4, 21/01/2005, rio Mamoã(MZUESC 532).

Distribuição: Atlântico Ocidental - Bermuda, Flórida, Golfo do México, América Central, Antilhas, norte da América do Sul e Brasil (do Pará até Santa Catarina) (Melo 1996).
Notas ecológicas: Em praias lodosas e principalmente em manguezais. Vivem em galerias parcialmente cheias de água e sob pedras na marca da maré alta e também no interior de troncos apodrecidos. Do entre-marés até pequenas profundidades (Melo 1996).

\section{Hexapanopeus caribbaeus (Stimpson, 1871)}

Material examinado ( $\mathrm{N}=69)$ : $\mathrm{N}=3,18 / 09 / 2004$, rio Santana, Est. 02 (MZUESC 497); N=4, 18/09/2004, rio Santana, Est. 04 (MZUESC 499); N=4, 18/09/2004, rio Cachoeira, Est. 06 (MZUESC 500); N=3, 18/09/2004, rio Santana, Est. 04 (MZUESC 583); N=38, 24/09/2004, rio Cachoeira, Est. 04 (MZUESC 596); N=3, 13/01/2005, rio Santana, Est. 04 (MZUESC 608); N=12, 13/01/2005, rio Cachoeira, Est. 06 (MZUESC 612); N=1, 13/01/2005, rio Cachoeira, Est. 07 (MZUESC 616); N=1, 13/01/2005, rio Cachoeira, Est. 08 (MZUESC 621).

Distribuição: Atlântico Ocidental - Antilhas, norte da América do Sul e Brasil (do Pará até o Rio Grande do Sul) (Melo 1996, Rieger et al 1996).

Notas ecológicas: Ocorre em fundos arenosos desde o entre-marés até 55 m (Melo 1996).

Panopeus lacustris Desbone, 1867

Material examinado $(\mathrm{N}=24)$ : $\mathrm{N}=2,13 / 03 / 2003$, rio Cachoeira, Av. 02 de Julho (MZUESC 45); N=1, 20/02/2001, 
rio Cachoeira, Av. Lomanto Júnior (MZUESC 46); N=2, 23/ 04/2003, rio Acuípe (MZUESC 77); N=1, 01/05/2003, rio Acuípe, Est. 02 (MZUESC 81); N=3, 16/07/2003, rio Cururupe (MZUESC 236); N=3, 04/03/2004, rio Acuípe, Est. 04 (MZUESC 337); N=4, 21/01/2005, rio Mamoã (MZUESC 534); $\mathrm{N}=4$, 22/02/2005, rio Almada, Est. 03 (MZUESC 556); $\mathrm{N}=2$, 21/08/2004, rio Cachoeira, praia da Maramata (MZUESC 577); $\mathrm{N}=1,18 / 09 / 2004$, rio Santana, Est. 03 (MZUESC 578); $\mathrm{N}=1$, 13/01/2005, rio Santana (MZUESC 605).

Distribuição: Atlântico Ocidental - Bermuda, Flórida, Antilhas, Colômbia e Brasil (do Pará ao Rio de Janeiro) (Melo 1996, Barros et al 1997a).

Notas ecológicas: Vive sob pedras, em estuários, baías e canais, podendo ser encontrada, ainda, em locais poluídos, sob galhos em putrefação e associada a ostras (Melo 1996).

Comentários: Espécie citada previamente para Ilhéus por Melo (1985), como P. herbstii.

\section{Panopeus occidentalis Saussure, 1857}

Material examinado ( $N=13)$ : $N=2,30 / 09 / 2003$, rio Acuípe (MZUESC 400); N=6, 21/01/2005, rio Mamoã (MZUESC 533); N=1, 18/09/2004, rio Santana, Est. 03 (MZUESC 579); N=3, 24/09/2004, rio Cachoeira, Est. 04 (MZUESC 595); N=1, 24/09/2004, rio Cachoeira, Est. 08 (MZUESC 603).

Distribuição: Atlântico Ocidental - costa leste dos EUA, América Central, Antilhas, norte da América do Sul e Brasil (de Pernambuco ao Rio Grande do Sul) (Melo 1996).

Notas ecológicas: Em fundos de areia, rocha e cascalho, entre algas e raízes do mangue, do entre-marés até 20 m (Calado \& Sousa 2003).

\section{Panopeus rugosus A. Milne Edwards, 1880}

Material examinado ( $\mathrm{N}=11)$ : $\mathrm{N}=2,01 / 05 / 2003$, rio Acuípe, Est. 02 (MZUESC 80); N=1, 21/05/2003, Pque Mun. Boa Esperança (MZUESC 123); N=1, 14/11/2003, rio Almada (MZUESC 257); N=1, 30/09/2003, rio Acuípe (MZUESC 399); $\mathrm{N}=2$, 22/02/2005, rio Almada, Est. 05 (MZUESC 561); $\mathrm{N}=2$, 24/09/2004, rio Cachoeira, Est. 03 (MZUESC 591); N=1, 24/ 09/2004, rio Cachoeira, Est. 04 (MZUESC 594); N=1, 13/01/ 2005, rio Cachoeira, Est. 04 (MZUESC 606).

Distribuição: Atlântico Ocidental - Flórida, Golfo do México, América Central, Antilhas, norte da América do Sul, Guianas e Brasil (de Pernambuco até o Rio Grande do Sul) (Melo 1996).

Notas ecológicas: Em fundos de areia, conchas, rochas e corais (Melo 1996), e em substrato de lama, entre as raízes do mangue (Calado \& Sousa 2003).

Comentários: Espécie citada previamente para Ilhéus por Melo (1985).

\section{Superfamília Ocypodoidea Rafinesque, 1815}

Família Ocypodidae Rafinesque, 1815

Ocypode quadrata (Fabricius, 1787)

Material examinado ( $\mathrm{N}=3$ ): $\mathrm{N}=2,03 / 03 / 2004$, rio Sargi (MZUESC 323); N=1, 21/01/2005, rio Mamoã (MZUESC 542).

Distribuição: Atlântico Ocidental - Bermuda, Flórida, Golfo do México, América Central, Antilhas, norte da América do Sul, Guianas e Brasil (Fernando de Noronha e do Pará até o Rio Grande do Sul) (Melo 1996).

Notas ecológicas: Em praias arenosas, do supralitoral até a área de dunas. Jovens constróem galerias próximas do nível da maré alta máxima e junto à vegetação da praia. Os jovens são diurnos e os adultos noturnos (Melo 1996).

\section{Uca leptodactyla Rathbun, 1898}

Material examinado ( $\mathrm{N}=233$ ): $\mathrm{N}=7,27 / 02 / 2003$, rio Cachoeira, Av. Sapetinga (MZUESC 30); N=36, 13/03/2003, rio Cachoeira, Praia do Cristo (MZUESC 38); N=12, 23/04/ 2003, rio Acuípe, Est. 06, foz (MZUESC 73); N=19, 23/04/ 2003, rio Acuípe, Est. 06, foz (MZUESC 74); N=9, 01/05/ 2003, rio Acuípe, Est. 02 (MZUESC 89); N=55, 21/05/2003, Pque Mun. Boa Esperança (MZUESC 130); N=4, 01/05/2003, rio Acuípe (MZUESC 139); N=7, 04/06/2003, rio Acuípe, Est. 05(MZUESC 150); N=18, 16/07/2003, rio Cururupe(MZUESC 223); $\mathrm{N}=10,03 / 03 / 2004$, rio Sargi (MZUESC 321); $N=2$, 21/ 08/2004, rio Cachoeira, praia da Maramata (MZUESC 494); $\mathrm{N}=19,21 / 01 / 2005$, rio Mamoã (MZUESC 544); $\mathrm{N}=35,22 / 02 /$ 2005, rio Almada, Est. 05 (MZUESC 565).

Distribuição: Atlântico Ocidental - Flórida, Golfo do México, Antilhas, Venezuela e Brasil (do Maranhão até Santa Catarina) (Melo 1996, Calado \& Sousa 2003).

Notas ecológicas: Ocorre no supralitoral e no entremarés. Em águas altamente salinas. Prefere substrato arenoso, com pouca ou nenhuma mistura de lama. Algumas populações ocupam ambientes lamosos, perto de árvores do mangue, mas, quase sempre, nas margens de grandes baías ou ilhas expostas ao mar aberto (Melo 1996, Calado \& Sousa 2003).

Uca maracoani (Latreille, 1802-1803)

Material examinado (N=6): $\mathrm{N}=1,04 / 06 / 2003$, rio Acuípe, Est. 05 (MZUESC 145); N=3, 04/07/2003, rio Acuípe, Est. 05 (MZUESC 200); N=1, 04/03/2004, rio Acuípe, Est. 04 (MZUESC 403); $\mathrm{N}=1,22 / 02 / 2005$, rio Almada, Est. 05 (MZUESC 564).

Distribuição: Atlântico Ocidental - Antilhas, Venezuela, Guianas e Brasil (do Pará até o Paraná) (Melo 1996, Barros et al 1997a).

Notas ecológicas: Ao longo de margens de baías calmas. Ocorre sempre ao nível da maré vazante máxima, em substratos lodosos, perto de árvores de mangue, vivendo em regimes de salinidade marinha polialino e mesoalino (Melo 1996, Calado \& Sousa 2003). 
Comentários: Espécie citada previamente para Ilhéus por Melo (1985).

Uca mordax (Smith, 1870)

Material examinado ( $\mathrm{N}=32)$ : $\mathrm{N}=17,22 / 02 / 2005$, rio Almada, Est. 06 (MZUESC 570); N=15, 22/02/2005, rio Almada, Est. 07 (MZUESC 573).

Distribuição: Atlântico Ocidental - Golfo do México, América Central, norte da América do Sul, Guianas e Brasil (do Pará até São Paulo) (Melo 1996).

Notas ecológicas: Em margens de riachos e manguezais. As populações com maior número de indivíduos se estabelecem acima do nível dos manguezais, onde a água é praticamente doce (Melo 1996), fato este que foi observado no local de coleta dos espécimes.

\section{Uca rapax (Smith, 1870)}

Material examinado ( $N=69$ ): $N=43,21 / 05 / 2003$, Pque Mun. Boa Esperança (MZUESC 124); N=3, 01/05/2003, rio Acuípe, Est. 02 (MZUESC 136); N=7, 01/05/2003, rio Acuípe (MZUESC 137); N=9, 14/11/2003, rio Almada (MZUESC 255); $\mathrm{N}=2,04 / 12 / 2003$, rio Almada (MZUESC 391); $\mathrm{N}=4,18 / 09$ / 2004, rio Santana (MZUESC 496); N=1, 09/02/2005, rio Mamoã (MZUESC 546).

Distribuição: Atlântico Ocidental - Flórida, Golfo do México, Antilhas, Venezuela e Brasil (do Pará até Santa Catarina) (Melo 1996).

Notas ecológicas: Vivem em galerias escavadas no lodo ou areia lamosa na vizinhança dos manguezais (Melo 1996).

\section{Uca thayeri Rathbun, 1900}

Material examinado ( $N=128)$ : $N=15,23 / 04 / 2003$, rio Acuípe, Est. 01 (MZUESC 67); N=3, 23/04/2003, rio Acuípe, Est. 06, foz (MZUESC 72); N=6, 01/05/2003, rio Acuípe, Est. 02 (MZUESC 84); N=4, 21/05/2003, Pque Mun. Boa Esperança (MZUESC 129); N=3, 01/05/2003, rio Acuípe (MZUESC 138); $\mathrm{N}=22,04 / 06 / 2003$, rio Acuípe, Est. 05 (MZUESC 148); N=3, 04/07/2003, rio Acuípe, Est. 05 (MZUESC 199); N=12, 16/07/ 2003, rio Cururupe (MZUESC 222); N=4, 30/09/2003, rio Acuípe (MZUESC 243); N=5, 30/09/2003, rio Acuípe, Est. 05 (MZUESC 245); $\mathrm{N}=1,14 / 11 / 2003$, rio Almada (MZUESC 258); $\mathrm{N}=1,03 / 03 / 2004$, rio Sargi (MZUESC 322); N=10, 04/03/2004, rio Acuípe, Est. 04 (MZUESC 339); N=6, 04/12/2003, rio Almada (MZUESC 388); N=9, 18/09/2004, rio Santana (MZUESC 428); N=9, 21/01/2005, rio Mamoã (MZUESC 545); $\mathrm{N}=6,22 / 02 / 2005$, rio Almada, Est. 03 (MZUESC 559); N=9, 22/02/2005, rio Almada, Est. 05 (MZUESC 566).

Distribuição: Atlântico Ocidental - Flórida, Golfo do México, Antilhas, Guatemala, Panamá, Venezuela e Brasil (do Maranhão até Santa Catarina) (Melo 1996).
Notas ecológicas: Em regiões lamacentas da periferia de manguezais, muitas vezes, na área sombreada (Melo 1996).

\section{Uca vocator (Herbst, 1804)}

Material examinado (N=3): N=2, 14/11/2003, rio Almada (MZUESC 251); N=1, 04/12/2003, rio Almada (MZUESC 387).

Distribuição: Atlântico Ocidental - Golfo do México, América Central, Antilhas, norte da América do Sul, Guianas e Brasil (de Pernambuco até Santa Catarina) (Melo 1996).

Notas ecológicas: Em estuários, por vezes ocorrendo no sedimento que fica entre as árvores do manguezal. Também encontrada rio acima nos limites do mangue (Melo 1996).

\section{Ucides cordatus (Linnaeus, 1763)}

Material examinado ( $\mathrm{N}=23)$ : $\mathrm{N}=1,27 / 02 / 2003$, rio Cachoeira, Av. Sapetinga (MZUESC 08); N=2, 23/04/2003, rio Acuípe, Est. 01 (MZUESC 68); N=5, 21/05/2003, Pque Mun. Boa Esperança (MZUESC 128); N=9, 04/12/2003, rio Almada (MZUESC 389); N=1, 04/03/2004, rio Acuípe, Est. 04 (MZUESC 402); $\mathrm{N}=1,21 / 01 / 2005$, rio Mamoã (MZUESC 543); $\mathrm{N}=2,22 / 02 / 2005$, rio Almada, Est. 05 (MZUESC 567); $\mathrm{N}=1$, 22/02/2005, rio Almada, Est. 06 (MZUESC 571); N=1, 22/02/ 2005, rio Almada, Est. 07 (MZUESC 574).

Distribuição: Atlântico Ocidental - Flórida, Golfo do México, América Central, Antilhas, norte da América do Sul, Guianas e Brasil (do Pará até Santa Catarina) (Melo 1996).

Notas ecológicas: Em ambientes pantanosos, entre raízes de mangue. Constrói galerias, retas e rasas, em áreas de água salobra (Melo 1996).

\section{Superfamília Grapsoidea MacLeay, 1838}

Família Gecarcinidae MacLeay, 1838

Cardisoma guanhumi Latreille, 1825

Material examinado ( $N=2)$ : N=1, 21/05/2003, Pque Mun. Boa Esperança (MZUESC 127); N=1, 17/02/2005, rio Cachoeira, Banco da Vitória (MZUESC 465).

Distribuição: Atlântico Ocidental - Bermuda, Flórida, Golfo do México, Antilhas, Colômbia, Venezuela e Brasil (do Ceará até São Paulo) (Melo 1996).

Notas ecológicas: Espécie encontrada no supralitoral dos canais estuarinos, em tocas no substrato areno-lamoso. É semi-terrestre e gregária, de hábito comumente noturno (Melo 1996, Calado \& Sousa 2003). Habitações observadas no supralitoral de todos os estuários amostrados. Alguns espécimes avistados na abertura ou próximo à abertura de suas tocas, construídas em terreno firme, em porção do rio sem influência da maré.

Comentários: Espécie previamente citada para Ilhéus por Melo (1985). 
Família Grapsidae MacLeay, 1838

Cyclograpsus integer (H. Milne-Edwards, 1837)

Material examinado (N=1): N=1, 21/01/2005, rio Mamoã (MZUESC 535).

Distribuição: Atlântico Ocidental - Flórida, Golfo do México, América Central, norte da América do Sul e Brasil (do Ceará até Santa Catarina). Atlântico Oriental - de Cabo Verde até Senegal. Indo-Pacífico (Melo 1996).

Notas ecológicas: Vivem em galerias, em áreas marinhas pantanosas. Também no supra e mesolitoral de praias rochosas. Pode também ocorrer em estuários e arrecifes (Melo 1996). O único exemplar foi coletado no supralitoral, em terreno arenoso.

\section{Goniopsis cruentata (Latreille, 1803)}

Material examinado ( $N=34)$ : $N=2,27 / 02 / 2003$, rio Cachoeira, Av. Sapetinga (MZUESC 31); N=5, 23/04/2003, rio Acuípe, Est. 01 (MZUESC 66); N=6, 01/05/2003, rio Acuípe, Est. 02 (MZUESC 83); N=2, 01/05/2003, rio Acuípe, Est. 03 (MZUESC 94); N=4, 21/05/2003, Pque Mun. Boa Esperança (MZUESC 131); N=1, 04/07/2003, rio Acuípe, Est. 05 (MZUESC 198); N=3, 16/07/2003, rio Cururupe (MZUESC 219); N=1, 30/09/2003, rio Acuípe (MZUESC 244); $\mathrm{N}=1,14 / 11 / 2003$, rio Almada (MZUESC 259); $\mathrm{N}=1,03 / 03$ / 2004, rio Sargi (MZUESC 315); N=1, 04/03/2004, rio Acuípe, Est. 04 (MZUESC 404); N=2, 18/09/2004, rio Santana (MZUESC 453); N=1, 21/01/2005, rio Mamoã (MZUESC 536); $\mathrm{N}=1,22 / 02 / 2005$, rio Almada, Est. 02 (MZUESC 552); $\mathrm{N}=3$, 22/02/2005, rio Almada, Est. 05 (MZUESC 563).

Distribuição: Atlântico Ocidental - Bermuda, Flórida, Golfo do México, Antilhas, Guianas e Brasil (Fernando de Noronha, Atol das Rocas e do Pará até Santa Catarina) (Melo 1996, Targino et al 2001).

Notas ecológicas: Em manguezais, sob as raízes ou troncos das árvores. Em praias lodosas, em braços de mar ou estuários. Do supralitoral até o entre-marés (Melo 1996).

\section{Pachygrapsus gracilis (Saussure, 1859)}

Material examinado ( $N=95)$ : $N=5,27 / 02 / 2003$, rio Cachoeira, Av. Sapetinga (MZUESC 29); N=2, 13/03/2003, rio Cachoeira, Av. 02 de Julho (MZUESC 44); N=4, 23/04/ 2003, rio Acuípe (MZUESC 76); N=25, 01/05/2003, rio Acuípe, Est. 02 (MZUESC 87); N=5, 21/05/2003, Pque Mun. Boa Esperança (MZUESC 135); N=7, 04/06/2003, rio Acuípe, Est. 05 (MZUESC 147); N=1, 04/07/2003, rio Acuípe, Est. 05 (MZUESC 206); $\mathrm{N}=1,16 / 07 / 2003$, rio Cururupe (MZUESC 220); N=5, 30/09/2003, rio Acuípe, Est. 05 (MZUESC 246); $\mathrm{N}=1,14 / 11 / 2003$, rio Almada (MZUESC 260); $\mathrm{N}=1,03 / 03$ / 2004, rio Sargi (MZUESC 316); N=1, 04/03/2004, rio Acuípe, Est. 04 (MZUESC 340); N=3, 04/12/2003, rio Almada (MZUESC 396); N=2, 18/09/2004, rio Cachoeira, Est. 06 (MZUESC 427); N=1, 18/09/2004, rio Santana(MZUESC 429);
$\mathrm{N}=2,18 / 09 / 2004$, rio Santana, Est. 03 (MZUESC 430); N=3, 24/09/2004, rio Cachoeira, Est. 04 (MZUESC 431); N=10, 21/ 01/2005, rio Mamoã (MZUESC 537); N=8, 22/02/2005, rio Almada, Est. 03 (MZUESC 557); N=5, 22/02/2005, rio Almada, Est. 05 (MZUESC 562); N=3, 24/09/2004, rio Cachoeira, Est. 04(MZUESC 597).

Distribuição: Atlântico Ocidental - Carolina do Norte, Flórida, Golfo do México, Antilhas, norte da América do Sul, Guianas e Brasil (Ilha da Trindade e do Pará até o Rio Grande do Sul). Atlântico Oriental - de Cabo Verde até Angola. Mar Mediterrâneo. Pacífico Leste - da Califórnia ao Peru (Melo 1996, Barros et al 1997a).

Notas ecológicas: Em áreas rochosas, no entre-marés. Sob pedras e pilares de embarcadouros. Ocasionalmente vive entre as raízes do mangue e em praias arenosas. Também em estuários e recifes (Melo 1996). Em Ilhéus foi encontrada freqüentemente associada a troncos em decomposição.

\section{Pachygrapsus transversus (Gibbes, 1850)}

Material examinado ( $\mathrm{N}=11)$ : $\mathrm{N}=9,21 / 08 / 2004$, rio Cachoeira, praia da Maramata (MZUESC 418); N=2, 22/02/ 2005, rio Almada, Est. 02 (MZUESC 548).

Distribuição: Atlântico Ocidental - Bermuda, da Carolina do Norte até a Flórida, Golfo do México, Antilhas, norte da América do Sul e Brasil (Trindade e do Ceará até o Rio Grande do Sul) e Uruguai. Atlântico Oriental - de Cabo Verde até Angola. Mar Mediterrâneo. Pacífico Leste - Califórnia até o Peru (Melo 1996).

Notas ecológicas: No entre-marés, sob pedras. Em pilares de embarcadouros e em praias arenosas. Também entre raízes do mangue (Melo 1996).

\section{Família Sesarmidae Dana, 1851}

Aratus pisonii (H. Milne Edwards, 1837)

Material examinado ( $\mathrm{N}=16)$ : $\mathrm{N}=4,23 / 04 / 2003$, rio Acuípe, Est. 01 (MZUESC 70); N=1, 01/05/2003, rio Acuípe, Est. 02 (MZUESC 85); N=1, 21/05/2003, Pque Mun. Boa Esperança (MZUESC 132); N=2, 14/11/2003, rio Almada, (MZUESC 254); N=3, 03/03/2004, rio Sargi (MZUESC 317); $\mathrm{N}=4$, 21/01/2005, rio Mamoã (MZUESC 540); $\mathrm{N}=1,13 / 01$ / 2005, rio Cachoeira, Est. 08 (MZUESC 620).

Distribuição: Atlântico Ocidental - Flórida, Golfo do México, Antilhas, norte da América do Sul, Guianas e Brasil (do Pará até São Paulo). Pacífico Leste - da Nicarágua até o Peru (Melo 1996, Barros et al 1997a).

Notas ecológicas: Em estuários, sobre rochas ou em pilares de embarcadouros. Comum em manguezais, onde escala as árvores (Melo 1996).

Armases angustipes (Dana, 1852) 
Material examinado $(\mathrm{N}=26)$ : $\mathrm{N}=5,04 / 06 / 2003$, rio Acuípe, Est. 04 (MZUESC 144); N=1, 04/03/2003, Pque Mun. Boa Esperança (MZUESC 248); N=3, 14/11/2003, rio Almada (MZUESC 256); N=3, 04/03/2004, rio Acuípe, Est. 04 (MZUESC 341); N=5, 04/12/2003, rio Almada (MZUESC 394); $\mathrm{N}=9,21 / 01 / 2005$, rio Mamoã (MZUESC 539).

Distribuição: Atlântico Ocidental - México, Antilhas e Brasil (do Pará até Santa Catarina) (Melo 1996, Barros et al 1997a).

Notas ecológicas: É encontrado ao lado de Sesarma rectum, nos mesmos tipos de habitats estuarinos, sendo mais freqüente na região marginal (Melo 1996).

\section{Armases rubripes (Rathbun, 1897)}

Material examinado (N=6): N=1, 21/05/2003, Pque Mun. Boa Esperança (MZUESC 186); N=2, 04/12/2003, rio Almada (MZUESC 392); N=1, 21/01/2005, rio Mamoã (MZUESC 538); N=1, 22/02/2005, rio Almada, Est. 03 (MZUESC 558); N=1, 22/02/2005, rio Almada, Est. 07 (MZUESC 572).

Distribuição: Atlântico Ocidental - América Central, norte da América do Sul, Guianas, Brasil (do Ceará ao Rio Grande do Sul), Uruguai e Argentina (Melo 1996).

Notas ecológicas: Ocorre em pântanos salgados, no sedimento entre as raízes. Também em fissuras e cavidades de trapiches e rochas (Melo 1996). Alguns espécimes do rio Almada foram coletados acima da linha d’água em porção do rio já sem influência da maré.
Sesarma curacaoense De Man, 1892 (Figura 9)

Material examinado $(\mathrm{N}=34)$ : $\mathrm{N}=1,04 / 06 / 2003$, rio Acuípe, Est. 05 (MZUESC 146); N=1, 16/07/2003, rio Cururupe (MZUESC 221); N=1, 30/09/2003, rio Acuípe, Est. 05 (MZUESC 247); N=5, 14/11/2003, rio Almada (MZUESC 252); $N=1,04 / 12 / 2003$, rio Almada (MZUESC 397); $N=25,21 /$ 01/2005, rio Mamoã (MZUESC 541).

Distribuição: Atlântico Ocidental - Flórida, Antilhas (Cuba, Porto Rico, Jamaica, Curaçao), Trinidad e Tobago, Panamá e Brasil (até a Bahia) (Abele 1992).

Notas ecológicas: Ocorre no solo dos manguezais e em aglomerados de ostras e rochas em substrato de lama (Abele 1992).

\section{Sesarma rectum Randall, 1840}

Material examinado $(\mathrm{N}=26)$ : $\mathrm{N}=15,23 / 04 / 2003$, rio Acuípe, Est. 01 (MZUESC 69); N=5, 01/05/2003; rio Acuípe, Est. 02 (MZUESC 88); N=5, 01/05/2003, rio Acuípe, Est. 03 (MZUESC 95); N=4, 22/05/2003, Pque Mun. Boa Esperança (MZUESC 126); N=1, 04/12/2003, rio Almada (MZUESC 395); $\mathrm{N}=1,22 / 02 / 2005$, rio Almada, Est. 06 (MZUESC 568).

Distribuição: Atlântico Ocidental - Granada (Antilhas), Venezuela, Guianas e Brasil (do Amapá até Santa Catarina) (Melo 1996, Schubart et al 1999).

Notas ecológicas: Escava tocas que ficam a descoberto vários dias, de acordo com altura da preamar. Pode ser encontrado em todos os regimes de salinidade (Melo 1996).

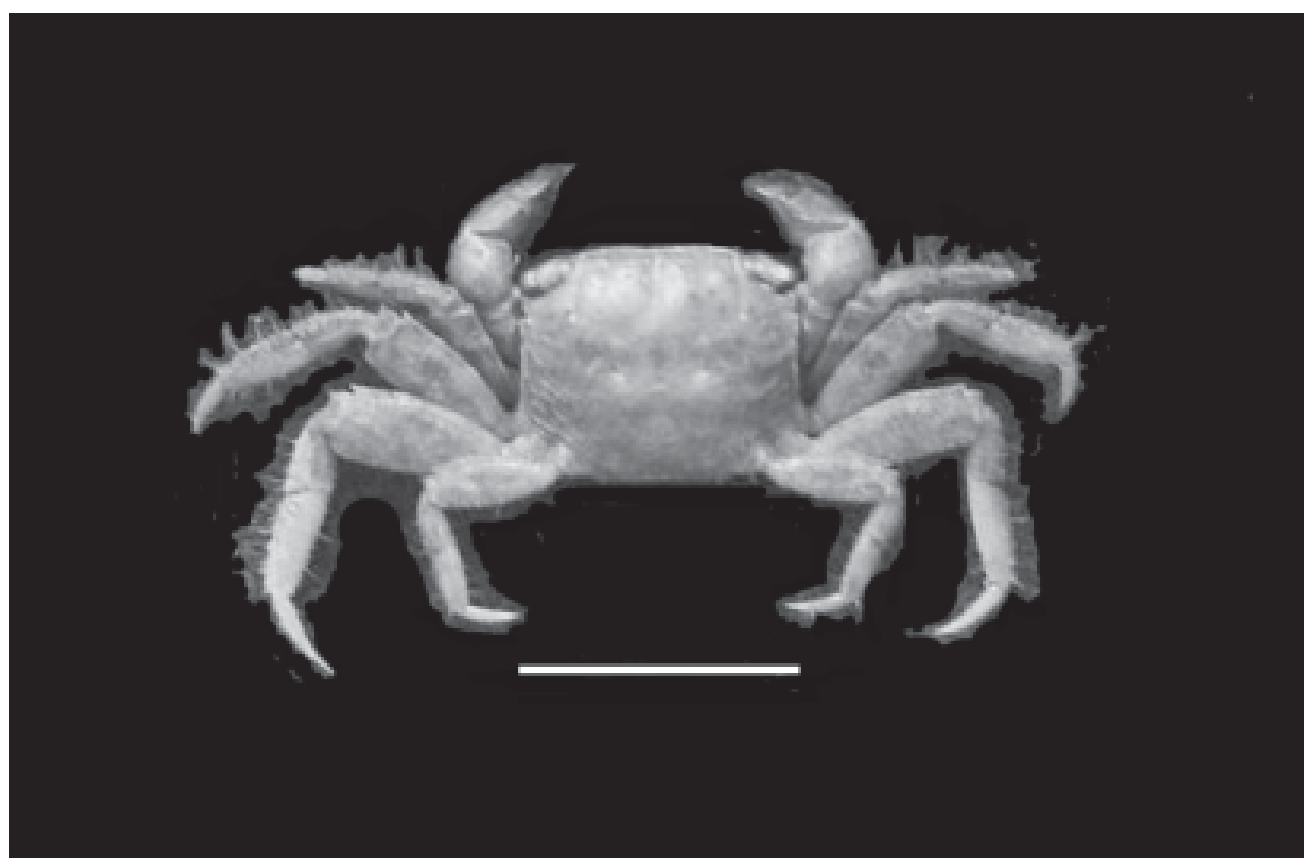

Figura 9. Sesarma curacaoense De Man do estuário do rio Almada, Ilhéus, Brasil. Barra = $10 \mathrm{~mm}$.

Figure 9. Sesarma curacaoense De Man from the Almada River estuary, Ilhéus, Brazil. Scale bar = $10 \mathrm{~mm}$. 


\section{Discussão}

\section{Distribuição Geográfica das Espécies}

A grande maioria das espécies aqui citadas apresenta ampla distribuição latitudinal no Atlântico Ocidental. Das 51 espécies consideradas, seis têm distribuição Circumtropical (Alpheus bouvieri, Cyclograpsus integer, Pachygrapsus gracilis, P. transversus, Petrolisthes armatus e Synalpheus fritzmuelleri), duas Anfi-Atlântica (Alpheus intrinsecus e A. pontederiae), duas são Anfi-Americanas (Alpheus estuariensis e Aratus pisonii) e uma espécie introduzida Indo-Pacífica (Charybdis hellerii), no sentido de Melo (1985). As demais espécies são Atlânticas Ocidentais.

Alpheus heterochaelis, cujo limite meridional de distribuição conhecido para o Atlântico Ocidental era até então o Estado de Alagoas (Christoffersen 1984, Calado \& Sousa 2003), tem, com sua ocorrência em Ilhéus, seu limite meridional de distribuição ampliado. Esta espécie tem ocorrência registrada no Brasil, no Pará (Christoffersen 1984), Maranhão (Ramos-Porto et al 1978), Paraíba (Christoffersen 1984), Pernambuco (Coelho et al 2002) e Alagoas (Sousa et al 2000). As freqüentes identificações errôneas de $A$. heterochaelis teriam obscurecido o fato de que $A$. armillatus é a espécie de Alpheus mais comum nos ambientes estuarinos da costa do Brasil, onde ocorre predominância de substrato duro na região entre-marés (Christoffersen 1984). Apesar da semelhança, A. heterochaelis pode ser distinguido de $A$. armillatus, por não apresentar espinho agudo mésio-distal na porção flexora do mero dos quelípodos, sendo que a quela menor do macho dessa espécie possui forma do tipo “balaeniceps” (Chace Jr 1972, Christoffersen 1984).

Merguia rhizophorae foi descrita por Rathbun (1900) a partir de material coletado pela Expedição Branner-Agassiz no rio Paraíba do Norte, Paraíba. A espécie é endêmica do Nordeste brasileiro, ocorrendo nos Estados do Piauí (Christoffersen 1998), Ceará e Rio Grande do Norte (Fausto Filho 1968), Paraíba (Rathbun 1900), Pernambuco (Coelho 1965/66) e Alagoas (Sousa et al 2000), sendo este último o limite austral de distribuição conhecido para a espécie na costa Atlântica Ocidental. Com a ocorrência da espécie em Ilhéus, amplia-se a distribuição meridional conhecida para M. rhizophorae na costa Atlântica da América do Sul.

Abele (1992) em sua revisão do gênero Sesarma nas Américas, examinou material de $S$. curacaoense proveniente da Flórida, Caribe e da Bahia. O primeiro registro da espécie no Brasil foi o de Rathbun (1918), tendo sido posteriormente registrada em Pernambuco (Coelho \& Ramos 1972) e Rio Grande do Norte (Ferreira \& Sankarankutty 2002). As raras coletas no país talvez se devam, em parte, a uma possível confusão com a espécie $S$. crassipes, conforme apontado por Abele (1992). De acordo com o autor, o status de $S$. curacaoense e S. crassipes no Brasil requer uma revisão. S. crassipes, cuja localidade-tipo é Pernambuco, seria uma espécie restrita ao Caribe e os registros brasileiros pertenceriam a S. curacaoense. Rathbun (1918) e Abele (1992) referem-se à Bahia como limite meridional de distribuição conhecido para $S$. curacaoense na costa Atlântica da América do Sul. O material desse Estado, estudado por ambos, depositado na coleção do $U$. S. National Museum, foi coletado entre 1876 e 1877, na localidade de Mapelle, Bay of Bahia. Por certo os autores referiram-se à Mapele, localidade situada no atual município de Simões Filho (1247’S; 38²3’W), leste da Baía de Todos os Santos, Bahia. Assim, o registro de S. curacaoense representa uma ampliação da distribuição meridional para a espécie, embora de pequena magnitude. É possível que futuras coletas ao sul de Ilhéus revelem uma distribuição geográfica mais ampla, dado o desconhecimento em relação à fauna da região.

\section{A Ocorrência de Charybdis hellerii}

O rio Cachoeira é a terceira localidade na Bahia com ocorrência documentada do siri exótico Charybdis hellerii. Estudos preliminares detectaram um aumento da população da espécie na Baía de Todos os Santos, provavelmente fruto da competição por alimento e habitat com os portunídeos nativos, da inexistência de predadores naturais e do não aproveitamento do siri pela pesca artesanal (C.R.G. Carqueija, comunicação pessoal).

O primeiro exemplar de C. hellerii da Baía de Camamu, situada ao sul da Baía de Todos os Santos, foi coletado no ano 2000 (Almeida et al 2003). Posteriormente, o primeiro autor examinou exemplares provenientes de amostragens periódicas realizadas no local desde 2002, sugerindo que a espécie encontra-se estabelecida na região.

Em Ilhéus, onde foram realizadas amostragens exaustivas ao longo de mais de três anos de trabalho, foi coletado apenas um exemplar juvenil de C. hellerii. Este fato leva a crer que a colonização das águas locais esteja ainda em fase inicial. É possível que $C$. helleri tenha chegado na região por dispersão via estágios larvais a partir de localidades situadas no litoral norte da Bahia ou, ainda, que tenha chegado através de água de lastro descarregada por navios no porto de Ilhéus (1447'S; 3902’W). Tal mecanismo é tido como um dos principais meios de introdução de espécies exóticas de crustáceos (Tavares \& Mendonça Jr 2004).

Uma lista de registros prévios desta espécie na costa brasileira pode ser verificada em Bezerra \& Almeida (2005).

\section{Riqueza de Espécies}

A riqueza da fauna carcinológica de um estuário pode se avaliada comparando-se o número total de espécies com outros estuários (Coelho \& Coelho-Santos 1990). CoelhoSantos \& Coelho (2001) citaram 64 espécies de crustáceos 
decápodos para o estuário do rio Paripe, Ilha de Itamaracá, Pernambuco. Calado \& Sousa (2003) citaram 55 espécies para o complexo estuarino lagunar Mundaú-Manguaba, Alagoas. Ferreira \& Sankarankutty (2002) listaram 70 espécies para dois estuários do Rio Grande do Norte, sendo 52 para os estuários dos rios Casqueira e Conceição, e 49 para o do rio Potengi. Esses três trabalhos têm em comum a localização dos estuários estudados, todos no Nordeste brasileiro, e reúnem material coletado ao longo de vários anos de amostragem, sendo locais de fauna bem conhecida. Os números obtidos em nossa pesquisa são próximos àqueles de Alagoas (Calado \& Sousa 2003) e do Rio Grande do Norte (Ferreira \& Sankarankutty 2002). Essas diferenças em relação ao número de espécies devem-se provavelmente ao fato de que os estudos referidos acima apresentam um tempo de execução bem mais extenso.

Comparando o número de espécies de decápodos de Ilhéus com os de estuários situados no Pacífico Leste, a quantidade de espécies presentes em nossas amostragens foi superior a obtida por Hendrickx (1984) $(\mathrm{N}=31)$, para o Estero El Verde, Sinaloa, México, e é bastante próxima daquela de Echeverría-Saenz et al (2003), que listaram 52 espécies para o sistema de manguezais Térraba-Sierpe, na Costa Rica, a principal área úmida da América Central.

Estas informações preenchem em parte a lacuna representada pela escassez de estudos de composição da fauna de decápodos do litoral sudeste da Bahia. Os dados gerados poderão direcionar futuros trabalhos sobre a biologia e ecologia da fauna de crustáceos da região. Além disso, fica demonstrado que as áreas amostradas apresentam uma significativa riqueza de espécies, a qual se encontra ameaçada pela ação antrópica crescente. As informações aqui levantadas poderão constituir uma base útil para a realização de estudos sobre biodiversidade, além da elaboração de planos de manejo e conservação das áreas estudadas.

\section{Agradecimentos}

A UESC pelo financiamento do projeto "Inventariamento da Fauna de Crustáceos do Município de Ilhéus, Bahia - 00220.1100.337”. Ao Sr. Rui Rego Cavalcanti e equipe pelo apoio fundamental nas coletas de arrasto. Ao Dr. Christoph D. Schubart, University of Regensburg, Alemanha, pela identificação da espécie S. curacaoense. Aos colegas M. Sc. Sérgio M. Almeida e M. Sc. Luis Ernesto A. Bezerra (PPGO/UFPE) pelo registro fotográfico dos crustáceos e auxílio no tratamento das imagens, respectivamente. Aos professores Ronan X. Corrêa, Janisete G. S. Miller, Tania Barth e Binael S. Santos (in memorian) (DCB/UESC), e à Sra. Regina Farias (CEPEC/CEPLAC), pelo apoio em diversas etapas deste trabalho. Aos acadêmicos da UESC Joaldo R. Luz, Cynthia S. Soares e Ana Cácia F. Santos, pelo auxílio em campo e laboratório. A.O. de Almeida e P.A. Coelho agradecem, respectivamente, a FAPESB
(Fundação de Amparo à Pesquisa do Estado da Bahia) e ao CNPq pela concessão de bolsas de produtividade em pesquisa. J.T.A. dos Santos e N.R. Ferraz agradecem, respectivamente, a FAPESB e ao PROIIC/PIBIC/UESC pela concessão de bolsas de iniciação científica.

\section{Referências Bibliográficas}

ABELE, L.G. 1992. A review of the grapsid crab genus Sesarma (Crustacea: Decapoda: Grapsidae) in America, with the description of a new genus. Smithson. Contrib. Zool. 527:1-60.

ALMEIDA, A.O., COELHO, P.A. \& SANTOS, J.T.A. 2003. New records of decapod crustaceans (Dendrobranchiata and Brachyura) for the state of Bahia, Brazil. Nauplius 11(2):129-133.

BARROS, M.P. \& PIMENTEL, F.R. 2001. A fauna de Decapoda (Crustacea) do Estado do Pará, Brasil: lista preliminar de espécies. Bol. Mus. Para. Emílio Goeldi, sér. Zool. 17(1):15-41.

BARROS, M.P., PIMENTEL, F.R. \& SILVA, S.B. 1997a. Novos registros de Brachyura (Crustacea, Decapoda) para o litoral do Estado do Pará, Brasil. Nauplius 5(2):161-163.

BARROS, M.P., SILVA, S.B. \& PIMENTEL, F.R. 1997b. Ocorrência de Lysiosquilla scabricauda (Lamarck, 1818) (Stomatopoda, Lysiosquillidae) e Petrolisthes armatus (Gibbes, 1850) (Decapoda, Porcellanidae), no Estado do Pará, Brasil. Bol. Mus. Para. Emílio Goeldi, sér. Zool. 13(1):21-24.

BEZERRA, L.E.A. \& ALMEIDA, A.O. 2005. Primeiro registro da espécie Indo-Pacífica Charybdis hellerii (A. MilneEdwards, 1867) (Crustacea: Decapoda: Portunidae) para o litoral do Estado do Ceará. Tropical Oceanography 33(1):33-38.

BOND-BUCKUP, G. \& BUCKUP, L. 1989. Os Palaemonidae de águas continentais do Brasil meridional (Crustacea, Decapoda). Rev. Bras. Biol. 49(4):883-896.

CALADO, T.C.S. 2000. Registro de Callinectes sapidus Rathbun, 1896 (Crustacea Decapoda, Portunidae) na confluência dos rios Traipu e São Francisco (TraipuAlagoas). Bol. Estud. Ciênc. Mar 11:113-117.

CALADO, T.C.S. \& SOUSA, E.C. 2003. Crustáceos do complexo estuarino-lagunar Mundaú/Manguaba Alagoas. FAPEAL, Maceió.

CARQUEIJA, C.R.G. \& GOUVÊA, E.P. 1996. A ocorrência, na costa brasileira, de um Portunidae (Crustacea, Decapoda), originário do Indo-Pacífico e Mediterrâneo. Nauplius 4:105-112.

CHACE JR., F.A. 1972. The shrimps of the SmithsonianBredin Caribbean Expeditions with a summary of the West Indian shallow-water species (Crustacea: Decapoda: Natantia). Smithson. Contr. Zool. 98:1-179. 
CHRISTOFFERSEN, M.L. 1979. Decapod Crustacea: Alpheoida. Campagne de la Calypso au large des côtes atlantiques de l’Amérique du Sud (1961-1962). I. 36, Ann. Inst. océanogr, Monaco, Suppl. 55:297-377.

CHRISTOFFERSEN, M.L. 1984. The western Atlantic snapping shrimps related to Alpheus heterochaelis Say (Crustacea, Caridea), with the description of a new species. Pap. Avuls Zool., 35(19):189-208.

CHRISTOFFERSEN, M.L. 1998. Malacostraca. Eucarida. Caridea. Crangonoidea and Alpheoidea (Except Glyphocrangonidae and Crangonidae). In Catalogue of Crustacea of Brazil (P.S. Young, ed.). Museu Nacional, Rio de Janeiro, p.351-372.

COELHO, P.A. 1965/66. Os crustáceos decápodos de alguns manguezais de Pernambuco. Trab-s Inst. Oceanogr. Univ. Fed. Pe. 7/8:71-90.

COELhO, P.A. \& COELHO-SANTOS, M.A. 1990. Crustáceos decápodos e estomatópodos do rio Paripe, Itamaracá-PE. An. Soc. Nordest. Zool. 3:43-61.

COELHO, P.A. \& RAMOS, M.A. 1972. A constituição e a distribuição da fauna de decápodos do litoral leste da América do Sul entre as latitudes $5^{\circ} \mathrm{N}$ e $39^{\circ} \mathrm{S}$. Trab. Oceanogr. Univ. Fed. Pe. 13:133-236.

COELHO, P.A. \& RAMOS-PORTO, M. 1980. Crustáceos decápodos da costa do Maranhão, Brasil. Bolm. Inst. oceanogr. 29(2):135-138.

COELHO, P.A., RAMOS-PORTO, M. \& CALADO, T.C.S. 1986. Litoral do Rio Grande do Norte: Decapoda. Cad. ômega Univ. Fed. Rural PE, Sér. Ci. Aquát. 2:79-105.

COELHO, P.A., RAMOS-PORTO, M. \& MELO, G.A.S. 1990. Crustáceos decápodos do estado de Alagoas. An. Soc. Nordest. Zool. 3:21-34.

COELHO, P.A. \& SANTOS, M.C.F. 1995. Resultados das amostragens biológicas na pesca de camarões marinhos ao largo de Ilhéus, Ba. Bol. Téc. Cient. CEPENE 3(1):109-120.

COELHO, P.A., SANTOS, M.A.C., TORRES, M.F.A., MONTEIRO, B.R. \& ALMEIDA, V.A.K. 2002. Reino Animalia: Filo (ou Subfilo) Crustacea no Estado de Pernambuco. In Diagnóstico da biodiversidade de Pernambuco (M. Tabarelli \& J.M.C. Silva, eds.). Massangana, Recife, v. 2, p.429-482.

COELHO-SANTOS, M.A. \& COELHO, P.A. 2001. Crustacea Decapoda of the Paripe river estuary, Pernambuco, Brazil. Hydrobiologia 449(1-3):77-79.

D’INCAO, F. 1995. Taxonomia, padrões distribucionais e ecológicos dos Dendrobranchiata (Crustacea: Decapoda) do Brasil e Atlântico Ocidental. Tese de Doutorado, Universidade Federal do Paraná, Curitiba.

DINEEN, J.F., CLARK, P.F., HINES, A.H., REED, S.A. \& WALTON, H.P. 2001. Life history, larval description, and natural history of Charybdis hellerii (Decapoda, Brachyura, Portunidae), an invasive crab in the western Atlantic. J. Crust. Biol. 21 (3):774-805.
ECHEVERRÍA-SÁENZ, S., VARGAS, R. \& WEHRTMANN, I.S. 2003. Diversity of decapods inhabiting the largest mangrove system of Pacific Costa Rica. Nauplius 11(2):91-97.

FARIA-FILHO, A.F. \& ARAÚJO, Q.R. 2003. Zoneamento do meio físico do município de Ilhéus, Bahia, Brasil, utilizando a técnica de geoprocessamento. CEPLAC/ CEPEC. Boletim técnico nº 187, p.1- 20.

FAUSTO FILHO, J. 1968. Terceira contribuição ao inventário dos crustáceos decápodos marinhos do Nordeste Brasileiro. Arq. Ciênc. Mar 8(1):43-45.

FAUSTO FILHO, J. 1978. Os crustáceos estomatópodos e decápodos dos substratos de lama do Nordeste brasileiro. Arq. Ciênc. Mar 18(1/2):63-71.

FAUSTO FILHO, J. 1979. Os crustáceos estomatópodos e decápodos dos substratos de areia do Nordeste brasileiro. Arq. Ciênc. Mar 19(1/2):45-56.

FERREIRA, A.C. \& SANKARANKUTTY, C. 2002. Estuarine carcinofauna (Decapoda) of Rio Grande do Norte, Brazil. Nauplius 10(2):121-129.

HENDRICKX, M.E. 1984. Studies of the coastal marine fauna of southern Sinaloa, Mexico. II. The Decapod Crustaceans of Estero el Verde. An. Inst. Cienc. del Mar y Limnol., Univ. Autón, México 11(1):23-48.

HENDRICKX, M.E. 1995. Checklist of brachyuran crabs (Crustacea: Decapoda) from the eastern tropical Pacific. Bull. Inst. Royal Sci. Nat. Belgique, Biologie 65:125-150.

LEMAITRE, R. 1995. Charybdis helleri (Milne Edwards, 1867), a nonindigenous portunid crab (Crustacea: Decapoda: Brachyura) discovered in the Indian River lagoon system of Florida. Proc. Biol. Soc. Wash., 108(4):643-648.

MARTIN, J.W. \& DAVIS, G.E. 2001. An Updated Classification of the Recent Crustacea. Natural History Museum of Los Angeles County, Science Series, 39:1-124.

MARTÍNEZ-IGLESIAS, J.C., RÍOS, R. \& CARVACHO, A. 1996. Las especies del género Alpheus (Decapoda: Alpheidae) de Cuba. Rev. Biol. Trop. 44(3):401-429.

MELO, G.A.S. 1985. Taxonomia e padrões distribucionais e ecológicos dos Brachyura (Crustacea, Decapoda) do litoral sudeste do Brasil. Tese de doutorado, Universidade de São Paulo, São Paulo.

MELO, G.A.S. 1996. Manual de identificação dos Brachyura (caranguejos e siris) do litoral brasileiro. Editora Plêiade, São Paulo.

MELO, G.A.S. 1999. Manual de identificação dos Crustacea Decapoda do litoral brasileiro: Anomura, Thalassinidea, Palinuridea, Astacidea. Editora Plêiade, São Paulo.

MELO, G.A.S. 2003. Famílias Atyidae, Palaemonidae e Sergestidae. In Manual de identificação dos Crustacea Decapoda de água doce do Brasil (G.A.S. Melo, ed.). Edições Loyola, São Paulo, p.289-415. 
MIRANDA, E.E. \& COUTINHO, A.C. (Coord.). 2004. Brasil Visto do Espaço. Campinas: Embrapa Monitoramento por Satélite. Disponível em: < $\underline{\text { http:// }}$ www.cdbrasil.cnpm.embrapa.br>

ODUM, E.P. 1986. Ecologia. Guanabara Koogan, Rio de Janeiro.

RAMOS-PORTO, M. 1985/86. Revisão das espécies do gênero Leander E. Desmarest que ocorrem no litoral brasileiro. Trab. Oceanogr. Univ. Fed. Pe. 19:7-26.

RAMOS-PORTO, M. \& COELHO, P.A. 1990. Sinopse dos crustáceos decápodos brasileiros (família Palaemonidae). An. Soc. Nordest. Zool. 3(3):93-111.

RAMOS-PORTO, M., FERREIRA-CORREIA, M.M. \& SOUSA, N.R. 1978. Levantamento da fauna aquática da ilha de São Luís (Estado do Maranhão, Brasil). II. Crustacea. Bol. Lab. Hidrob. 2(1):77-88.

RATHBUN, M.J. 1900. The decapod and stomatopod Crustacea. I. Results of the Branner-Agassiz Expedition to Brazil. Proc. Wash. Acad. Sci. 2:133-156.

RATHBUN, M. J. 1918. The grapsoid crabs of America. Bull. U. S. natn. Mus. 97:1-461.

RIEGER, P. J., VIEIRA, R.R.R. \& SANTOS, S. 1996. Hexapanopeus caribbaeus (Stimpson, 1871) novo registro de Brachyura (Decapoda, Xanthidae) para o litoral do Rio Grande do Sul. Nauplius 4:169-170.

SANTOS, S., RIEGER, P.J., VIEIRA, R.R.R. \& BARUTOT, R.A. 2000. Composição e abundância dos Crustacea (Decapoda) na lagoa do Peixe, Rio Grande do Sul, Brasil. Revta bras. Zool. 17(1):213-223.

SCHUBART, C.D., HORST, D. \& DIESEL, R. 1999. First record of Sesarma rectum Randall (Brachyura, Sesarminae) from the lesser Antilles. Crustaceana 72(5):537-538.

SOUSA, E.C., COELHO, P.A. \& CALADO, T.C.S. 2000. Crustacea Decapoda dos canais da lagoa Manguaba no complexo estuarino-lagunar Mundaú-Manguaba, Alagoas, Brasil. Bol. Estud. Ciênc. Mar 11:165-192.

TARGINO, S.G, CAMPOS, C.E.C., SILVA, M.B., MELO, C.E.D.C.A \& MACÊDO, G.D. 2001. First record of the mangrove crab Goniopsis cruentata (Latreille, 1803) (Brachyura: Grapsidae) in Atol das Rocas, Brasil. Nauplius 9(2):169-170.

TAVARES, M. \& AMOUROUX, J.M. 2003, First record of the non-indigenous crab, Charybdis hellerii (A. MilneEdwards, 1867) from French Guyana (Decapoda, Brachyura, Portunidae). Crustaceana 76(5):625-630.

TAVARES, M. \& MENDONÇA JR, J.B. 2004. Introdução de Crustáceos Exóticos no Brasil: Uma Roleta Ecológica. In Água de Lastro e Bioinvasão (J. Silva \& R. Souza, eds). Interciência, Rio de Janeiro, p.59-76.

WILLLIAMS, A.B. 1974. The swimming crabs of the genus Callinectes (Decapoda, Portunidae). Fish. Bull. 72(3):685-798.
Título: Crustáceos decápodos estuarinos de Ilhéus, Bahia, Brasil.

Autores: Almeida, A.O., Coelho, P.A., Santos, J.T.A. and Ferraz, N.R.

Biota Neotropica, Vol. 6 ( número 2 ): 2006

http://www.biotaneotropica.org.br/v6n2/pt/ abstract?inventory+bn03406022006

Recebido em 10/11/05 - Versão reformulada recebida em 27/04/06 - Publicado em 19/07/2006

ISSN 1676-0603 
ANEXO - Espécies de crustáceos decápodos de Ilhéus, Bahia - distribuição por estação de coleta. APPENDIX - Decapod species from Ilhéus, State of Bahia, Brazil: distribution per sampling area.

I. Parque Municipal da Boa Esperança, ribeirão Iguape (14 47’01,3" S; 3903’ 49,6" W):

Alpheus pontederiae, Aratus pisonii, Armases angustipes, Armases rubripes, Cardisoma guanhumi, Goniopsis cruentata, Pachygrapsus gracilis, Panopeus rugosus, Sesarma rectum, Uca leptodactyla, Uca rapax, Uca thayeri, Ucides cordatus.

\section{Rio Acuípe}

Est. 01 (150 05' 19" S; 38 59' 56" W - próximo à entrada da Ilha dos Desejos): Aratus pisonii, Goniopsis cruentata, Sesarma rectum, Ucides cordatus, Uca thayeri.

Est. 02 (15² 05’ 21,8" S; 38 59’ 56,4" W - logo após ponte da rodovia BA-001): Alpheus pontederiae, Aratus pisonii, Callinectes danae, Eurytium limosum, Goniopsis cruentata, Pachygrapsus gracilis, Panopeus lacustris, Panopeus rugosus, Sesarma rectum, Uca leptodactyla, Uca rapax, Uca thayeri.

Est. 03 (15 04’ 53,6" S; 3900’13,8" W): Goniopsis cruentata, Macrobrachium acanthurus, Palaemon pandaliformis, Sesarma rectum.

Est. 04 (15²04’59,5" S; 38 59’ 56,0" W - antiga ponte sobre o rio Acuípe): Armases angustipes, Callinectes danae, Goniopsis cruentata, Pachygrapsus gracilis, Panopeus lacustris, Uca maracoani, Uca thayeri, Ucides cordatus.

Est. 05 (15²04’ 58,6" S; 38 59’ 53,4" W - passadiço de madeira sobre afluente do rio Acuípe): Alpheus pontederiae, Callinectes exasperatus, Eurytium limosum, Goniopsis cruentata, Pachygrapsus gracilis, Sesarma curacaoense, Uca leptodactyla, Uca maracoani, Uca thayeri.

Est. 06 (1505’41" S; 3859’50" W, foz): Callinectes danae, Uca leptodactyla, Uca thayeri.

\section{Rio Almada}

Est. 02 (14 46’27,2" S; 3903’ 14,8" W - foz do rio): Alpheus armillatus, Callinectes danae, Callinectes exasperatus, Clibanarius sclopetarius, Clibanarius vittatus, Goniopsis cruentata, Pachygrapsus transversus, Palaemon northropi.

Est. 03 (14 45’ 40,9" S; 39 03’ 39,4" W - bairro São Miguel): Armases rubripes, Pachygrapsus gracilis, Panopeus lacustris, Uca thayeri.

Est. 04 ( $14^{\circ}$ 45’ 07,5" S; 390 03’ 51,2" W - ponte da Rodovia Ilhéus-Itacaré): Macrobrachium acanthurus.

Est. 05 (14 43’30,3" S; 390 04’ 02,4" W): Goniopsis cruentata, Pachygrapsus gracilis, Panopeus rugosus, Uca leptodactyla, Uca maracoani, Uca thayeri, Ucides cordatus.

Est. 06 (14 41’ 06,5" S; 39॰ 04’ 32,0" W): Sesarma rectum, Uca mordax, Ucides cordatus.

Est. 07 (14 40’ 30,6" S; 390 04’ 43,5" W - ponto mais a montante): Armases rubripes, Uca mordax, Ucides cordatus.

\section{Rio Cachoeira}

Praia da Maramata (14 48’ 28,7" S; 3901’33,3" W): Alpheus bouvieri, Callinectes larvatus, Clibanarius antillensis, Clibanarius sclopetarius, Clibanarius vittatus, Eurypanopeus abbreviatus, Lepidophtalmus siriboia, Pachygrapsus transversus, Palaemon northropi, Panopeus lacustris, Petrolisthes armatus, Uca leptodactyla.

Praia do Cristo (14 48’ 22,9" S; 3901’ 55,0" W): Callinectes danae, Clibanarius sclopetarius, Clibanarius vittatus, Uca leptodactyla.

Av. 2 de Julho (14 48’ 05,4" S; $39^{\circ} 02^{\prime}$ 01,5" W): Callinectes exasperatus, Callinectes larvatus, Clibanarius sclopetarius, Pachygrapsus gracilis, Panopeus lacustris.

Av. Lomanto Júnior (14 48’31,1" S; 3902’08,3" W): Panopeus lacustris.

Av. Sapetinga (14 48’ 40,9" S; 3902’ 21,1" W): Callinectes danae, Callinectes exasperatus, Callinectes larvatus, Goniopsis cruentata, Pachygrapsus gracilis, Uca leptodactyla, Ucides cordatus.

Banco da Vitória (14 47’ 05,0" S; 39016’13,5" W): Cardisoma guanhumi. bocourti.

Arrasto, Est. 1 (14 47’28,8" S; 3905’ 45,3" W - próximo à estação de tratamento de esgotos de Ilhéus): Callinectes

Arrasto, Est. 2 (14 48’00,7" S; 3905’29,8" W - próximo à ilha de mangue): Callinectes danae, Callinectes sapidus.

http://www.biotaneotropica.org.br 
Arrasto, Est. 3 (14 47’ 57,9" S; 39² 4' 51,2" W): Callinectes bocourti, Callinectes danae, Panopeus rugosus, Farfantepenaeus sp.

Arrasto, Est. 4 (14 48’ 15,6"S; 39 04’ 22,3" W): Alpheus heterochaelis, Alpheus pontederiae, Callinectes danae, Farfantepenaeus sp, Hexapanopeus caribbaeus, Leander paulensis, Pachygrapsus gracilis, Panopeus occidentalis, Panopeus rugosus.

Arrasto, Est. 5 (14 48’ 51" S; 3903’27,4" W): Callinectes danae, Callinectes exasperatus, Callinectes sapidus, Farfantepenaeus sp.

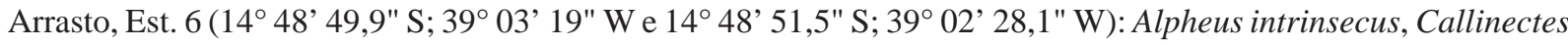
danae, Clibanarius vittatus, Farfantepenaeus sp, Hexapanopeus caribbaeus, Pachygrapsus gracilis.

Arrasto, Est. 7 (14 48’ 57,3"S; 39²02’30,5" W - Ponte Lomanto Júnior): Callinectes danae, Callinectes ornatus, Charybdis hellerii, Hexapanopeus caribbaeus, Leander paulensis.

Arrasto, Est. 8 (14 48’ 10" S; 39 02’ 12,3"W - em frente ao Bairro do Pontal): Acantholobulus schmitti, Alpheus armillatus, Alpheus intrinsecus, Aratus pisonii, Callinectes danae, Callinectes ornatus, Clibanarius vittatus, Farfantepenaeus sp, Hexapanopeus caribbaeus, Leander paulensis, Panopeus occidentalis, Synalpheus fritzmuelleri, Upogebia brasiliensis.

V. Rio Cururupe (14 52’51,0" S; 3901’34,9" W):

Eurytium limosum, Goniopsis cruentata, Pachygrapsus gracilis, Panopeus lacustris, Sesarma curacaoense, Uca leptodactyla, Uca thayeri, Upogebia brasiliensis.

VI. Rio Fundão, Est. 01 (14 48’ 00,1" S; 3903’31,8" W):

Callinectes danae.

VII. Rio Mamoã (14 35' 05,6" S; 39 03' 10,5"' W):

Alpheus estuariensis, Aratus pisonii, Armases angustipes, Armases rubripes, Callinectes exasperatus, Cyclograpsus integer, Eurytium limosum, Goniopsis cruentata, Merguia rhizophorae, Ocypode quadrata, Pachygrapsus gracilis, Panopeus lacustris, Panopeus occidentalis, Sesarma curacaoense, Uca leptodactyla, Uca rapax, Uca thayeri, Ucides cordatus.

VIII. Rio Santana:

$14^{\circ} 37^{\prime}$ 20,8" S; 3908’32,5"W: Goniopsis cruentata, Pachygrapsus gracilis, Panopeus lacustris, Uca thayeri.

$14^{\circ} 49^{\prime}$ 55,4"S; 3902' 55,3"W: Uca rapax.

Arrasto, Est. 01 (14 51’ 08,1" S; 3903’ 59,8" W): Callinectes bocourti.

Arrasto, Est. 02 (14 51’ 04" S; 39॰03’ 37,5" W): Hexapanopeus caribbaeus.

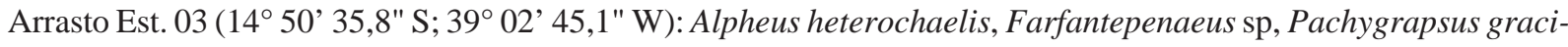
lis, Panopeus lacustris, Panopeus occidentalis, Upogebia brasiliensis.

Arrasto, Est. 04 (14 50’ 14,4" S ; 39 02’39,2" W): Callinectes bocourti, Callinectes danae, Callinectes exasperatus, Farfantepenaeus sp, Hexapanopeus caribbaeus, Leander paulensis.

IX. Rio Sargi (14 30’06,7" S ; 3902’ 29,4" W):

Aratus pisonii, Callinectes danae, Callinectes larvatus, Clibanarius sclopetarius, Eurytium limosum, Goniopsis cruentata, Ocypode quadrata, Pachygrapsus gracilis, Uca leptodactyla, Uca thayeri. 